\title{
ESTIMATING ADDITIVE CHARACTER SUMS FOR FUCHSIAN GROUPS
}

\author{
Dorian Goldfeld ${ }^{1}$, Cormac O’Sullivan
}

December, 2001

Dedicated to Robert Rankin, in memoriam

\section{Introduction.}

We begin with a brief review of some background material (see [Iw1], [Sh], for example, for more details). Let $\Gamma$ denote a Fuchsian group of the first kind, i.e., $\Gamma \leq P S L_{2}(\mathbb{R})$ acts discretely on the upper half plane $\mathfrak{H}=\{z \in \mathbb{C} \mid \operatorname{Im}(z)>0\}$, and every point on $\partial \mathfrak{H}$ is the limit of some orbit. The most important examples of such groups for arithmetic are the congruence groups. If we fix a fundamental domain $\mathfrak{F}$ for $\Gamma$ then $\mathfrak{F}$ will not, in general, be compact, and will have cusps $\mathfrak{a}, \mathfrak{b}, \mathfrak{c}, \ldots$ where the closure of $\mathfrak{F}$ meets $\hat{\mathbb{R}}=\mathbb{R} \cup\{\infty\}$. We assume, without loss of generality, that $\infty$ is one of these inequivalent cusps with stability subgroup $\Gamma_{\infty}=\left\{ \pm\left(\begin{array}{cc}1 & m \\ 0 & 1\end{array}\right) \mid m \in \mathbb{Z}\right\}$. At each other cusp a we introduce local coordinates via the scaling matrix $\sigma_{\mathfrak{a}} \in S L_{2}(\mathbb{R})$ so that $\sigma_{\mathfrak{a}} \infty=\mathfrak{a}$ and

$$
\Gamma_{\mathfrak{a}}=\{\gamma \in \Gamma \mid \gamma \mathfrak{a}=\mathfrak{a}\}=\sigma_{\mathfrak{a}} \Gamma_{\infty} \sigma_{\mathfrak{a}}{ }^{-1}
$$

We choose $\mathfrak{F}$ so that it contains the upper part of the vertical strip between $-1 / 2$ and $1 / 2$. Near each cusp $\mathfrak{a}$ the domain $\mathfrak{F}$ looks like the above strip scaled by $\sigma_{\mathfrak{a}}$.

Next, let $\chi \in \operatorname{Hom}\left(\Gamma, \mathbb{C}^{\times}\right)$and $\Lambda \in \operatorname{Hom}(\Gamma, \mathbb{C})$ be multiplicative and additive 'characters' for $\Gamma$. If $\chi$ and $\Lambda$ are trivial on $\Gamma_{\mathfrak{a}}$ then we can define the non-holomorphic Eisenstein series

$$
\begin{aligned}
& E_{\mathfrak{a}}(z, s, \chi)=\sum_{\gamma \in \Gamma_{\mathfrak{a}} \backslash \Gamma} \chi(\gamma) \operatorname{Im}\left(\sigma_{\mathfrak{a}}^{-1} \gamma z\right)^{s}, \quad z \in \mathfrak{H}, \\
& E_{\mathfrak{a}}(z, s, \Lambda)=\sum_{\gamma \in \Gamma_{\mathfrak{a}} \backslash \Gamma} \Lambda(\gamma) \operatorname{Im}\left(\sigma_{\mathfrak{a}}^{-1} \gamma z\right)^{s}, \quad z \in \mathfrak{H} .
\end{aligned}
$$

The series $E_{\mathfrak{a}}(z, s, \chi)$ with $|\chi|=1$ and $\chi$ trivial on all parabolic group elements has been understood since the work of Selberg [Se]. Initially defined for $\operatorname{Re}(s)>1$ it has a meromorphic continuation to all $s \in \mathbb{C}$. It satisfies a functional equation relating values at $s$ to those

\footnotetext{
${ }^{1}$ The first author is partially supported by a grant from the NSF.
} 
at $1-s$. In addition, for $\operatorname{Re}(s) \geq 1 / 2$ it is holomorphic in $s$ except perhaps for simple poles in $(1 / 2,1]$. If $\chi$ is trivial, then it has a simple pole at $s=1$ with residue $\operatorname{Vol}(\Gamma \backslash \mathfrak{H})^{-1}$. The importance of this series comes from, among other things, its role in the spectral theory of automorphic functions [Iw1] and its usefulness in deriving the analytic continuation of various $L$-functions.

The series $E_{\mathfrak{a}}(z, s, \Lambda)$ is less well understood and is the focus of this paper. We show that the series in definition (1.2) is absolutely convergent for $\operatorname{Re}(s)>3$. Note that while $E_{\mathfrak{a}}(z, s, \chi)$ satisfies the automorphy relation

$$
E_{\mathfrak{a}}(\gamma z, s, \chi)=\chi\left(\gamma^{-1}\right) E_{\mathfrak{a}}(z, s, \chi) \text { for all } \gamma \in \Gamma
$$

the corresponding relation with $\chi$ being replaced by $\Lambda$ is

$$
E_{\mathfrak{a}}(\gamma z, s, \Lambda)=E_{\mathfrak{a}}(z, s, \Lambda)+\Lambda\left(\gamma^{-1}\right) E_{\mathfrak{a}}(z, s) \quad \text { for all } \gamma \in \Gamma
$$

where $E_{\mathfrak{a}}(z, s)$ is the Eisenstein series with trivial character 1. They are, however, both eigenfunctions of the hyperbolic Laplacian $\Delta_{z}=y^{2}\left(\frac{\partial^{2}}{\partial x^{2}}+\frac{\partial^{2}}{\partial y^{2}}\right)$, for $z=x+i y$, with the same eigenvalue $-s(1-s)$.

Conjecture 1.1. The series $E_{\mathfrak{a}}(z, s, \Lambda)$ has an analytic continuation to $\operatorname{Re}(s) \geq 1-\epsilon$ for some $\epsilon>0$. In this region there is only a simple pole at $s=1$. For $|s-1|>\epsilon$ and $\operatorname{Re}(s) \geq 1-\epsilon$ we have $E_{\mathfrak{a}}(z, s, \Lambda) \ll(1+|\operatorname{Im}(s)|)^{C}$ for some constant $C=C_{\epsilon}<1$ where the implied constant may depend on $z, \Lambda$ and $\Gamma$.

Conjecture 1.1 is now known in many cases. We need to distinguish between those homomorphisms $\Lambda$ that are cuspidal, meaning they vanish on all parabolic group elements, and those that are non-cuspidal homomorphisms.

For $\Lambda$ cuspidal it has been shown in [Go1], [O'S1], [O'S2] that $E_{\mathfrak{a}}(z, s, \Lambda)$ has a meromorphic continuation to all $s$ in $\mathbb{C}$ with a simple pole at $s=1$. Furthermore, in this case, Petridis $[\mathrm{Pe}]$ has just recently proved that, for $z$ in a compact set, $E_{\mathfrak{a}}(z, s, \Lambda)$ is bounded on the vertical line $\operatorname{Re}(s)=\sigma$ for fixed $\sigma>1 / 2$, and $|\operatorname{Im}(s)|$ sufficiently large.

For $\Lambda$ non-cuspidal, we do not yet have these results. In section 6 we use the theory of Selberg-Kloosterman zeta functions to obtain the continuation of $E_{\mathfrak{a}}(z, s, \Lambda)$ slightly to the right of $\operatorname{Re}(s)=1$.

A simple example of a homomorphism $\Lambda$ in $\operatorname{Hom}(\Gamma, \mathbb{C})$ is the function that counts the appearances of a certain generator in reduced words of the group. More precisely let $\gamma_{1}, \gamma_{2}, \ldots, \gamma_{n}$ be a set of generators for $\Gamma$ and suppose $\gamma$ in $\Gamma$ equals a product of powers of these generators $\gamma_{i_{1}}^{e_{1}} \cdots \gamma_{i_{m}}^{e_{m}}$ for $e_{1}, \ldots, e_{m}$ in $\mathbb{Z}$. Then set

$$
\log _{\gamma_{j}}(\gamma)=\sum_{\substack{1 \leq k \leq m \\ i_{k}=j}} e_{k}
$$

This function is well defined provided it vanishes on the relators of the group, and it will then be a homomorphism from $\Gamma$ to $\mathbb{Z}$. 
Let $\Gamma=\Gamma_{0}(11) /\{ \pm 1\}$. In section 9 we will see that $\Gamma$ is generated by hyperbolic elements $A, B$ and parabolic elements $P_{0}, P_{\infty}$ satisfying the single relation $A B A^{-1} B^{-1} P_{0} P_{\infty}=1$. Explicitly we have

$$
A=\left(\begin{array}{cc}
-7 & -1 \\
22 & 3
\end{array}\right), B=\left(\begin{array}{cc}
4 & 1 \\
-33 & -8
\end{array}\right), P_{0}=\left(\begin{array}{cc}
1 & 0 \\
-11 & 1
\end{array}\right), P_{\infty}=\left(\begin{array}{ll}
1 & 1 \\
0 & 1
\end{array}\right)
$$

It may be seen that $\log _{A}$ and $\log _{B}$ are in $\operatorname{Hom}(\Gamma, \mathbb{Z})$ and we will show in section 9 that

$$
\operatorname{Res}_{s=1} E_{\infty}\left(z, s, \log _{A}\right)=\operatorname{Re}\left(C \int_{\infty}^{z} \eta(w)^{2} \eta(11 w)^{2} d w\right)
$$

where $\eta$ is Dedekind's eta function, and $C \approx 0.171+0.394 i$. As a consequence of theorem 7.3 (which utilizes Petridis' bound $[\mathrm{Pe}]$ ) we obtain ${ }^{2}$

Theorem 1.2. Let $\Gamma=\Gamma_{0}(11) /\{ \pm 1\}, A=\left(\begin{array}{cc}-7 & -1 \\ 22 & 3\end{array}\right), C \approx 0.171+0.394 i$. Then for fixed $z \in \mathfrak{H}$ and $T \rightarrow \infty$

$$
\sum_{\substack{a b \\
c d \\
|c z+d|^{2} \leq T}} \log _{A}\left(\left(\begin{array}{ll}
a & b \\
c & d
\end{array}\right)\right)=\frac{T}{y} \operatorname{Re}\left(C \int_{\infty}^{z} \eta(w)^{2} \eta(11 w)^{2} d w\right)+O\left(T^{\frac{1}{2}+\epsilon}\right) .
$$

This example is an illustration of the more general case where $\Gamma$ is a congruence group and $\Lambda$ is cuspidal. We have been able to derive formulas similar to that given in theorem 1.2 with the product $\eta(w)^{2} \eta(11 w)^{2}$ being replaced by a suitable modular form for the group (see theorem 7.3). If $\Gamma$ is not a congruence group or $\Lambda$ is not cuspidal, we can still prove some weaker statements (see theorem 7.2).

Let $\mathcal{S}_{2}(\Gamma)$ denote the space of holomorphic cusp forms of weight 2 for $\Gamma$. If $f(z) \in \mathcal{S}_{2}(\Gamma)$ then $f(z) d z$ is a holomorphic differential form on $\Gamma \backslash \mathfrak{H}$ and we define

$$
\langle\gamma, f\rangle=\int_{z}^{\gamma z} f(w) d w
$$

to be a modular symbol. It does not depend on $z \in \mathfrak{H}$ and clearly $\Lambda_{f}=\langle\cdot, f\rangle \in \operatorname{Hom}(\Gamma, \mathbb{C})$. For each cusp $\mathfrak{a}$ we have $\Lambda_{f}(\gamma)=0$ for all $\gamma \in \Gamma_{\mathfrak{a}}$ by [Kna, proposition 11.1]. This shows that

\footnotetext{
${ }^{2}$ Since writing this paper an error has been found in Petridis' bound. The correct result [Ri], [Pe-Ri] proves $E_{\mathfrak{a}}(z, s, \Lambda)$ has polynomial growth in $|\operatorname{Im}(s)|$ for $1 / 2<\operatorname{Re}(s)$ and $z$ in a compact set. Let $\psi$ be a smooth, compactly supported function on $\mathbb{R}^{+}$. By standard contour integration techniques, (see [Ch-O'S] for example)

$$
\sum_{\substack{a b \\
c d}} \log _{A} \begin{array}{lll}
\begin{array}{l}
a \\
c
\end{array} & b
\end{array} \quad \psi\left(\frac{|c z+d|^{2}}{T}\right)=\frac{T}{y} \operatorname{Re} C \int_{\infty}^{z} \eta(w)^{2} \eta(11 w)^{2} d w \int_{0}^{\infty} \psi(r) d r+O\left(T^{\frac{1}{2}+\epsilon}\right)
$$

With the appropriate choice of $\psi$ theorems 1.2 and 7.3 may be recovered but with error term $O\left(T^{1-\epsilon}\right)$, see [Ri], [Pe-Ri].
} 
modular symbols are cuspidal. In the next section we see that modular symbols generate all cuspidal homomorphisms.

It has been established in [Go1], [O'S1] that for these special homomorphisms (modular symbols) the series (1.2) is absolutely convergent for $\operatorname{Re}(s)>2$, has a meromorphic continuation to all $s \in \mathbb{C}$, and a functional equation similar to that of $E_{\alpha}(z, s, \chi)$. The continuation is obtained by extending a method of Selberg based on Fredholm theory. These results are expanded upon by Petridis [Pe] using the technique of expressing $E_{\alpha}(z, s, \Lambda)$ as linear combinations of

$$
\left.\frac{d}{d \epsilon} E_{\alpha}\left(z, s, \chi_{\epsilon}\right)\right|_{\epsilon=0}
$$

for $\chi_{\epsilon}(\gamma)=e^{i \epsilon \Lambda_{j}(\gamma)}$ with $\Lambda_{j}$ the real and imaginary parts of $\Lambda, j=0,1$. Further references are [Di-O'S], [Go2].

\section{Basic properties.}

Let $\Gamma$ be a Fuchsian group of the first kind acting on $\mathfrak{H}$. Its elements are classified as hyperbolic, parabolic or elliptic according to their fixed points in $\mathfrak{H} \cup \hat{\mathbb{R}}$. Hyperbolic elements have two fixed points on $\hat{\mathbb{R}}$, parabolic elements have just one and elliptic elements have one fixed point in $\mathfrak{H}$.

If $\Gamma \backslash \mathfrak{H}$ has genus $g, r$ elliptic fixed points in $\Gamma \backslash \mathfrak{H}$ and $m$ cusps (the parabolic fixed points) on $\Gamma \backslash \hat{\mathbb{R}}$ then by a result from $[\mathrm{F}-\mathrm{K}]$, (see also [Iw2]), we can describe $\Gamma$ as a group in terms of $2 g+r+m$ primitive elements. Explicitly there are $2 g$ hyperbolic elements $A_{i}, B_{i}, 1 \leq i \leq g$, $r$ elliptic elements $E_{j}, 1 \leq j \leq r$ and $m$ parabolic elements $P_{l}, 1 \leq l \leq m$ that generate $\Gamma$. For $[A, B]=A B A^{-1} B^{-1}$ the relations are

$$
\left[A_{1}, B_{1}\right] \ldots\left[A_{g}, B_{g}\right] E_{1} \ldots E_{r} P_{1} \ldots P_{m}=1, E_{j}^{e j}=1
$$

for $1 \leq j \leq r$ and integers $e_{j} \geq 2$.

Set $\mathcal{V}(\Gamma)=\operatorname{Hom}(\Gamma, \mathbb{C})$ the $\mathbb{C}$-vector space of functions $\Lambda: \Gamma \rightarrow \mathbb{C}$ satisfying $\Lambda\left(\gamma_{1} \gamma_{2}\right)=$ $\Lambda\left(\gamma_{1}\right)+\Lambda\left(\gamma_{2}\right)$ for all $\gamma_{1}, \gamma_{2} \in \Gamma$. Denote the subspace of functions that are zero on $\Gamma_{\mathfrak{a}}$ by $\mathcal{V}_{\mathfrak{a}}(\Gamma)$ and set

$$
\mathcal{W}(\Gamma)=\bigcap_{\mathfrak{a}} \mathcal{V}_{\mathfrak{a}}(\Gamma)
$$

the cuspidal homomorphisms. We have seen that $\Lambda_{f} \in \mathcal{W}(\Gamma)$ for $f \in \mathcal{S}_{2}(\Gamma)$. On our set of generators $\Lambda \in \mathcal{V}(\Gamma)$ must satisfy $\Lambda\left(E_{j}\right)=0$ and $\sum_{l} \Lambda\left(P_{l}\right)=0$. Thus the dimensions of $\mathcal{V}(\Gamma), \mathcal{V}_{\mathfrak{a}}(\Gamma)$ and $\mathcal{W}(\Gamma)$ are $2 g+m-1,2 g+m-2$ and $2 g$, respectively, for $m \geq 2$. For $m=0,1$ they all have dimension $2 g$.

Let $\mathcal{M}_{2}(\Gamma)$ denote the space of holomorphic, weight 2, modular forms for $\Gamma$. As before we call the subspace of cusp forms $\mathcal{S}_{2}(\Gamma)$. From [Sh, theorems 2.23, 2.24] we have $\operatorname{dim}\left(\mathcal{S}_{2}(\Gamma)\right)=$ $g$ and $\operatorname{dim}\left(\mathcal{M}_{2}(\Gamma)\right)=g$ if $m=0$ and $g+m-1$ otherwise.

Now, we shall restrict our attention to Fuchsian groups of the first kind with at least one cusp, i.e., $m \geq 1$. For $\Lambda \in \mathcal{V}_{\mathfrak{a}}(\Gamma)$ we define $E_{\mathfrak{a}}(z, s, \Lambda)$ using (1.2). A fundamental question arises: where does this series converge? To answer this question it seems necessary to express 
$\Lambda$ in terms of integrals of modular forms around closed curves in $\Gamma \backslash \mathfrak{H}$. If $f(z) \in \mathcal{M}_{2}(\Gamma)$ then it has a Fourier expansion at each cusp a, namely

$$
j\left(\sigma_{\mathfrak{a}}, z\right)^{-2} f\left(\sigma_{\mathfrak{a}} z\right)=\sum_{n=0}^{\infty} c_{\mathfrak{a}}(n) e^{2 \pi i n z} .
$$

If each $c_{\mathfrak{a}}(0)=0$ then $f$ is a cusp form. The space $\mathcal{M}_{2}(\Gamma)=\mathcal{S}_{2}(\Gamma) \oplus \mathcal{E}_{2}(\Gamma)$ where $\mathcal{E}_{2}(\Gamma)$ is generated by Eisenstein series. In fact we may choose a basis $g_{1}, \ldots, g_{m-1}$ for $\mathcal{E}_{2}(\Gamma)$ where each $g_{i}$ has constant term $c_{\mathfrak{a}}(0)=1$ at one cusp $\mathfrak{a}$, constant term $c_{\mathfrak{b}}(0)=-1$ at another cusp $\mathfrak{b}$ and zero constant terms at all remaining cusps. We will prove this in section 4 .

In what follows we extend the definition of modular symbol (1.5) to include integrals of modular forms, not just cusp forms. For $g \in \mathcal{M}_{2}(\Gamma)$ define $\langle\gamma, g\rangle=\int_{z}^{\gamma z} g(w) d w$. As before, this makes sense for $z \in \mathfrak{H}$ and is independent of the base point $z$.

Proposition 2.1. Let $f_{1}, \ldots f_{g}$ be a basis for $\mathcal{S}_{2}(\Gamma)$ and $g_{1}, \ldots g_{m-1}$ the basis for $\mathcal{E}_{2}(\Gamma)$ described above (we require the constant terms in the Fourier expansion of each $g_{j}$ to be real at each cusp). Define $A_{i}, B_{i}, C_{j} \in \mathcal{V}(\Gamma)$ as follows

$$
A_{i}(\gamma)=\operatorname{Im}\left\langle\gamma, f_{i}\right\rangle, B_{i}(\gamma)=\operatorname{Re}\left\langle\gamma, f_{i}\right\rangle, C_{j}(\gamma)=\operatorname{Re}\left\langle\gamma, g_{j}\right\rangle
$$

for $1 \leq i \leq g$ and $1 \leq j \leq m-1$. Then this is a basis for $\mathcal{V}(\Gamma)$.

ProOf: Since the dimension of $\mathcal{V}(\Gamma)$ is $2 g+m-1$ we need only prove these elements are linearly independent. Suppose, to the contrary, that

$$
\left(\sum a_{i} A_{i}+\sum b_{i} B_{i}+\sum c_{j} C_{j}\right)(\gamma)=0
$$

for all $\gamma$ in $\Gamma$. By considering the real and imaginary parts of the above sum separately we may assume $a_{i}, b_{i}, c_{j} \in \mathbb{R}$. By setting $q_{a}(w)=\sum a_{i} f_{i}(w), q_{b}(w)=\sum b_{i} f_{i}(w)$ and $q_{c}(w)=\sum c_{j} g_{j}(w)$ we see that $\operatorname{Im}\left\langle\gamma, q_{a}\right\rangle+\operatorname{Re}\left\langle\gamma, q_{b}\right\rangle+\operatorname{Re}\left\langle\gamma, q_{c}\right\rangle=0$. Now for $\gamma_{\mathfrak{b}}$ generating $\Gamma_{\mathfrak{b}}$ we have $\operatorname{Im}\left\langle\gamma_{\mathfrak{b}}, q_{a}\right\rangle+\operatorname{Re}\left\langle\gamma_{\mathfrak{b}}, q_{b}\right\rangle=0$, therefore, $\operatorname{Re}\left\langle\gamma_{\mathfrak{b}}, q_{c}\right\rangle=0$ and the real constant term of $q_{c}$ in its Fourier expansion at $\mathfrak{b}$ must be zero. Repeating this argument at each cusp we find that $q_{c} \in \mathcal{S}_{2}(\Gamma)$ and hence $c_{j}=0$ for each $j$. We are left with $\operatorname{Im}\left\langle\gamma, q_{a}\right\rangle+\operatorname{Re}\left\langle\gamma, q_{b}\right\rangle=0$. Set $q=-i q_{a}+q_{b}$ so that $\operatorname{Re}\langle\gamma, q\rangle=0$ for all $\gamma \in \Gamma$. This implies that $q=0$, (see [Kna, prop. 11.13], for example). Therefore, $i q_{a}=q_{b}$ and since $a_{i}, b_{i}$ are real they must all be zero.

We deduce that for any $\Lambda \in \mathcal{V}(\Gamma)$ there exist $p$ in $\mathcal{M}_{2}(\Gamma)$ and $q$ in $\mathcal{S}_{2}(\Gamma)$ so that

$$
\Lambda(\gamma)=\langle\gamma, p+q\rangle+\overline{\langle\gamma, p-q\rangle}
$$

Thus we have proven a special case of the Eichler-Shimura isomorphism

$$
\mathcal{M}_{2}(\Gamma) \oplus \mathcal{S}_{2}(\Gamma) \cong \operatorname{Hom}(\Gamma, \mathbb{C})
$$

as $\mathbb{R}$-vector spaces. For simplicity we set $p_{\Lambda}=p+q$ and $q_{\Lambda}=p-q$ then

$$
\Lambda(\gamma)=\left\langle\gamma, p_{\Lambda}\right\rangle+\overline{\left\langle\gamma, q_{\Lambda}\right\rangle}, \text { with } p_{\Lambda}, q_{\Lambda} \in \mathcal{M}_{2}(\Gamma)
$$


If $\Lambda \in \mathcal{W}(\Gamma)$ then we have $p_{\Lambda}, q_{\Lambda} \in \mathcal{S}_{2}(\Gamma)$. Also if $\Lambda \in \mathcal{V}_{\mathfrak{a}}(\Gamma)$ then we can choose $p_{\Lambda}, q_{\Lambda}$ with zero constant terms in their Fourier expansion at $\mathfrak{a}$. This means that we can define

$$
P_{\mathfrak{a}}(z, \Lambda)=\int_{\mathfrak{a}}^{z} p_{\Lambda}(w) d w, \quad Q_{\mathfrak{a}}(z, \Lambda)=\int_{\mathfrak{a}}^{z} q_{\Lambda}(w) d w
$$

If $j\left(\sigma_{\mathfrak{b}}, z\right)^{-2} p_{\Lambda}\left(\sigma_{\mathfrak{b}} z\right)=\sum_{n=0}^{\infty} p_{\mathfrak{b}}(n) e^{2 \pi i n z}$, then

$$
\begin{aligned}
P_{\mathfrak{a}}\left(\sigma_{\mathfrak{a}} z, \Lambda\right) & =\int_{\sigma_{\mathfrak{a}}-1 \mathfrak{a}}^{z} j\left(\sigma_{\mathfrak{a}}, w\right)^{-2} p_{\Lambda}\left(\sigma_{\mathfrak{a}} w\right) d w \\
& =\int_{\infty}^{z} \sum_{n=1}^{\infty} p_{\mathfrak{a}}(n) e^{2 \pi i n w} d w \\
& =\frac{1}{2 \pi i} \sum_{n=1}^{\infty} \frac{p_{\mathfrak{a}}(n)}{n} e^{2 \pi i n z}
\end{aligned}
$$

Similarly $Q_{\mathfrak{a}}\left(\sigma_{\mathfrak{a}} z, \Lambda\right)=\frac{1}{2 \pi i} \sum_{n=1}^{\infty} \frac{q_{\mathfrak{a}}(n)}{n} e^{2 \pi i n z}$. Suppose that the Fourier coefficients of $p_{\Lambda}$, $q_{\Lambda}$ satisfy

$$
p_{\mathfrak{a}}(n), q_{\mathfrak{a}}(n) \ll n^{R_{\Lambda}}
$$

for some $R_{\Lambda}$ then

$$
\begin{aligned}
2 \pi i \Lambda(\gamma)= & 2 \pi i\left(P_{\mathfrak{a}}(\gamma z)-P_{\mathfrak{a}}(z)+\overline{Q_{\mathfrak{a}}(\gamma z)-Q_{\mathfrak{a}}(z)}\right) \\
= & \sum_{n>0} \frac{p_{\mathfrak{a}}(n)}{n}\left(e^{2 \pi i n \sigma_{\mathfrak{a}}{ }^{-1} \gamma z}-e^{2 \pi i n \sigma_{\mathfrak{a}}-1} z\right) \\
& \quad+\sum_{n>0} \frac{\overline{q_{\mathfrak{a}}(n)}}{n}\left(e^{-2 \pi i n \overline{\sigma_{\mathfrak{a}}-1} \gamma z}-e^{-2 \pi i n \overline{\sigma_{\mathfrak{a}}-1} z}\right) \\
\ll & \sum_{n \neq 0}|n|^{R_{\Lambda}-1}\left(e^{-2 \pi|n| \operatorname{Im}\left(\sigma_{\mathfrak{a}}^{-1} \gamma z\right)}+e^{-2 \pi|n| \operatorname{Im}\left(\sigma_{\mathfrak{a}}{ }^{-1} z\right)}\right) \\
\ll & \operatorname{Im}\left(\sigma_{\mathfrak{a}}{ }^{-1} \gamma z\right)^{-\left(R_{\Lambda}+\varepsilon\right)}+\operatorname{Im}\left(\sigma_{\mathfrak{a}}^{-1} z\right)^{-\left(R_{\Lambda}+\varepsilon\right)} .
\end{aligned}
$$

Therefore, if $\operatorname{Re}(s)=\sigma$,

$$
\begin{aligned}
E_{\mathfrak{a}}(z, s, \Lambda) & =\sum_{\gamma \in \Gamma_{\mathfrak{a}} \backslash \Gamma} \Lambda(\gamma) \operatorname{Im}\left(\sigma_{\mathfrak{a}}{ }^{-1} \gamma z\right)^{s} \\
& \ll \sum_{\gamma \in \Gamma_{\mathfrak{a}} \backslash \Gamma} \operatorname{Im}\left(\sigma_{\mathfrak{a}}{ }^{-1} \gamma z\right)^{\sigma-R_{\Lambda}-\varepsilon}+\operatorname{Im}\left(\sigma_{\mathfrak{a}}{ }^{-1} z\right)^{-R_{\Lambda}-\varepsilon} \sum_{\gamma \in \Gamma_{\mathfrak{a}} \backslash \Gamma} \operatorname{Im}\left(\sigma_{\mathfrak{a}}{ }^{-1} \gamma z\right)^{\sigma} \\
& =E_{\mathfrak{a}}\left(z, \sigma-R_{\Lambda}-\varepsilon\right)+\operatorname{Im}\left(\sigma_{\mathfrak{a}}{ }^{-1} z\right)^{-R_{\Lambda}-\varepsilon} E_{\mathfrak{a}}(z, \sigma),
\end{aligned}
$$

which is absolutely convergent for $\sigma>1+R_{\Lambda}+\varepsilon$ and $\sigma>1$. So we have proved the following result. 
Theorem 2.2. For $\Lambda \in \mathcal{V}_{\mathfrak{a}}(\Gamma)$ define $p_{\Lambda}, q_{\Lambda}$ as in $(2.2)$ and $R_{\Lambda}$ as in (2.4). Then $E_{\mathfrak{a}}(z, s, \Lambda)$ is absolutely convergent for $\operatorname{Re}(s)>\max \left(1, R_{\Lambda}+1\right)$.

If the $n$th Fourier coefficient (in the expansion at any cusp) of an element of some space $S$ is bounded by a constant times $n^{l}$ we will use the notation $\mathcal{B}(S) \leq l$.

Let $k$ be an even integral weight. When $\Gamma$ is a congruence group Deligne proved that

$$
\mathcal{B}\left(S_{k}(\Gamma)\right) \leq \frac{k-1}{2}+\epsilon .
$$

For the Eisenstein series in $\mathcal{E}_{k}(\Gamma)$ we can explicitly write their Fourier coefficients as divisor sums, see $[\mathrm{Sc}]$ and $[\mathrm{Sa}]$. Therefore, it can be shown that

$$
\mathcal{B}\left(\mathcal{E}_{k}(\Gamma)\right) \leq k-1+\epsilon .
$$

For $\Gamma$ a general Fuchsian group of the first kind we have Hardy's trivial bound

$$
\mathcal{B}\left(S_{k}(\Gamma)\right) \leq \frac{k}{2} .
$$

This has been improved by A. Good [Good] who obtained

$$
\mathcal{B}\left(S_{k}(\Gamma)\right) \leq \frac{k}{2}-\frac{1}{6}
$$

for $k>2$. The same bound should be valid for $k=2$ also. Finally, the analog of the trivial bound for elements of $\mathcal{E}_{k}(\Gamma)$ yields

$$
\mathcal{B}\left(\mathcal{E}_{k}(\Gamma)\right) \leq k .
$$

These cases for $k=2$ give us the next result.

Proposition 2.3. We have

$$
R_{\Lambda}=\left\{\begin{aligned}
\frac{1}{2}+\epsilon & \text { if } \Lambda \in \mathcal{W}(\Gamma), \Gamma \text { a congruence group } \\
1+\epsilon & \text { if } \Lambda \in \mathcal{V}(\Gamma), \Gamma \text { a congruence group } \\
1 & \text { if } \Lambda \in \mathcal{W}(\Gamma), \Gamma \text { a Fuchsian group of the first kind } \\
2 & \text { if } \Lambda \in \mathcal{V}(\Gamma), \Gamma \text { a Fuchsian group of the first kind, }
\end{aligned}\right.
$$

where the first two bounds are sharp.

Corollary 2.4. For any Fuchsian group $\Gamma$ and any $\Lambda \in V_{\mathfrak{a}}(\Gamma)$ the Eisenstein series $E_{\mathfrak{a}}(z, s, \Lambda)$ is absolutely convergent for $\operatorname{Re}(s)>3$. 
Proposition 2.5. For any $\Gamma$ and any positive even integer $k$ we have

$$
\mathcal{B}\left(\mathcal{M}_{k}(\Gamma)\right) \leq k
$$

In other words the nth Fourier coefficient of a modular form of weight $k$ is $\ll n^{k}$.

Proof: Let $\mathcal{F}_{\infty}=\left\{x+i y \in \mathfrak{H}\left|\frac{1}{2} \geq\right| x \mid\right\}$ and $\mathcal{F}$ the Ford fundamental domain for $\Gamma \backslash \mathfrak{H}$. Consequently, $\mathcal{F}=\left\{z \in \mathcal{F}_{\infty}|1<| j(\gamma, z) \mid\right.$ for all $\left.\gamma \in \Gamma-\Gamma_{\infty}\right\}$. By the Fourier expansion of $f$ in $\mathcal{M}_{k}(\Gamma)$ (similar to (2.1)) we see that there exists $C_{\Gamma}$ such that, for any $\mathfrak{a}$, $\left|f\left(\sigma_{\mathfrak{a}} z\right)\right| \leq C_{\Gamma} y^{k}$ as $y \rightarrow \infty$. Also, in a neighborhood of $\infty f$ is bounded. Thus, for $w \in \mathcal{F}$ we have

$$
f(w) \ll \max \left(1, \operatorname{Im}(w)^{-k}\right)
$$

for an implied constant depending only on $\Gamma$ and $f$.

Next, let $z$ be any element of $\mathcal{F}_{\infty}-\mathcal{F}$. There is a $\gamma \in \Gamma-\Gamma_{\infty}$ such that $z=\gamma w$ for $w \in \mathcal{F}$. By the construction of $\mathcal{F}$ we know that $\operatorname{Im}(z)<\operatorname{Im}(w)$. We may also bound $\operatorname{Im}(w)$ from above as follows. By [Kno, lemma 4] we have (for any $c, d \in \mathbb{R}$ )

$$
|c z+d|^{2} \geq\left(c^{2}+d^{2}\right) \frac{y^{2}}{1+4|z|^{2}} .
$$

Also, observe that since $\Gamma$ is discrete, the absolute values of bottom left entries of elements of $\Gamma-\Gamma_{\infty}$ are bounded from below, by $D_{\Gamma}$ say (see [Sh, Lemma 1.25]). Therefore,

$$
\operatorname{Im}(w)=\frac{y}{|j(\gamma, z)|^{2}} \leq \frac{y\left(1+4|z|^{2}\right)}{D_{\Gamma}^{2} y^{2}} \ll \frac{1}{y}
$$

since $|z|$ is bounded.

Noting that $y^{k / 2}|f(z)|$ is $\Gamma$ invariant we have, for $z$ in $\mathcal{F}_{\infty}-\mathcal{F}$,

$$
\begin{aligned}
y^{k / 2}|f(z)|=\operatorname{Im}(w)^{k / 2}|f(w)| & \ll \operatorname{Im}(w)^{k / 2} \max \left(1, \operatorname{Im}(w)^{-k}\right) \\
& \ll \max \left(\operatorname{Im}(w)^{k / 2}, \operatorname{Im}(w)^{-k / 2}\right) \\
& \ll y^{-k / 2} .
\end{aligned}
$$

This proves that $(2.5)$ is true for any $w$ in $\mathfrak{H}$. Then for $f(z)=\sum_{n=0}^{\infty} a_{n} e^{2 \pi i n z}$ we have

$$
a_{n}=e^{-2 \pi} \int_{0}^{1} f\left(x+\frac{i}{n}\right) e^{-2 \pi i n x} d x \ll n^{k}
$$

for $n \geq 1$. Finally, to show that the Fourier coefficients at another cusp $\mathfrak{a}$ have the same bound, use the fact that $j\left(\sigma_{\mathfrak{a}}, z\right)^{-k} f\left(\sigma_{\mathfrak{a}} z\right)$ is an element of $\mathcal{M}_{k}\left(\Gamma^{\prime}\right)$ for $\Gamma^{\prime}=\sigma_{\mathfrak{a}}{ }^{-1} \Gamma \sigma_{\mathfrak{a}}$ and apply the same reasoning. 


\section{Fourier expansions.}

We set $C_{\mathfrak{a} \mathfrak{b}}=\left\{c>0 \mid\left(\begin{array}{l}* * \\ c *\end{array}\right) \in \sigma_{\mathfrak{a}}{ }^{-1} \Gamma \sigma_{\mathfrak{b}}\right\}$ and label the elements of $\gamma$ by $\left(\begin{array}{ll}\gamma_{a} & \gamma_{b} \\ \gamma_{c} & \gamma_{d}\end{array}\right)$. For $c \in C_{\mathfrak{a} \mathfrak{b}}$ we define the Kloosterman sum

$$
S_{\mathfrak{a} \mathfrak{b}}(m, n, c)=\sum_{\substack{\gamma \in \Gamma_{\infty} \backslash \sigma_{\mathfrak{a}}{ }^{-1} \Gamma \sigma_{\mathfrak{b}} / \Gamma_{\infty} \\ \gamma_{c}=c}} e^{2 \pi i\left(n \frac{\gamma_{a}}{c}+m \frac{\gamma_{d}}{c}\right)} .
$$

We shall also need the sum

$$
S_{\mathfrak{a} \mathfrak{b}}(m, n, c, \Lambda)=\sum_{\substack{\gamma \in \Gamma_{\infty} \backslash \sigma_{\mathfrak{a}}{ }^{-1} \Gamma \sigma_{\mathfrak{b}} / \Gamma_{\infty} \\ \gamma_{c}=c}} \Lambda\left(\sigma_{\mathfrak{a}} \gamma \sigma_{\mathfrak{b}}{ }^{-1}\right) e^{2 \pi i\left(n \frac{\gamma_{a}}{c}+m \frac{\gamma_{d}}{c}\right)} .
$$

The Selberg-Kloosterman zeta function is defined as

$$
Z_{\mathfrak{a} \mathfrak{b}}(m, n, s)=\sum_{c \in C_{\mathfrak{a} \mathfrak{b}}} \frac{S_{\mathfrak{a} \mathfrak{b}}(m, n, c)}{c^{2 s}} .
$$

Replace $S_{\mathfrak{a} \mathfrak{b}}(m, n, c)$ by $S_{\mathfrak{a} \mathfrak{b}}(m, n, c, \Lambda)$ in $(3.3)$ to define $Z_{\mathfrak{a} \mathfrak{b}}(m, n, s, \Lambda)$. These functions occur naturally in the Fourier expansions of $E_{\mathfrak{a}}(z, s)$ and $E_{\mathfrak{a}}(z, s, \Lambda)$.

At each cusp $E_{\mathfrak{a}}\left(\sigma_{\mathfrak{b}}(z+1), s\right)=E_{\mathfrak{a}}\left(\sigma_{\mathfrak{b}}(z), s\right)$. By the Bruhat decomposition we have the following expansion

$$
E_{\mathfrak{a}}\left(\sigma_{\mathfrak{b}} z, s\right)=\delta_{\mathfrak{a} \mathfrak{b}} y^{s}+\phi_{\mathfrak{a} \mathfrak{b}}(s) y^{1-s}+\sum_{k \neq 0} \phi_{\mathfrak{a} \mathfrak{b}}(k, s) W_{s}(k z),
$$

where as before $z=x+i y, W_{s}(k z)$ is the Whittaker function $2 \sqrt{|k| y} K_{s-1 / 2}(2 \pi|k| y) e^{2 \pi i k x}$, and $K$ is the Bessel $K$-function. Also $\delta_{\mathfrak{a} \mathfrak{b}}=0$ unless $\mathfrak{a}=\mathfrak{b}$ when it is 1 . The coefficients $\phi_{\mathfrak{a} \mathfrak{b}}$ are given by

$$
\begin{aligned}
\phi_{\mathfrak{a} \mathfrak{b}}(s) & =\sqrt{\pi} \Gamma(s)^{-1} \Gamma\left(s-\frac{1}{2}\right) Z_{\mathfrak{a} \mathfrak{b}}(0,0, s), \\
\phi_{\mathfrak{a} \mathfrak{b}}(k, s) & =\pi^{s} \Gamma(s)^{-1}|k|^{s-1} Z_{\mathfrak{a} \mathfrak{b}}(k, 0, s) .
\end{aligned}
$$

See [Iw1] section 3.4 for these results.

As already mentioned, $E_{\mathfrak{a}}\left(\sigma_{\mathfrak{b}} z, s\right)$ has a simple pole at $s=1$ with residue $\operatorname{Vol}(\Gamma \backslash \mathfrak{H})^{-1}$. Therefore, when $k \neq 0, \phi_{\mathfrak{a} \mathfrak{b}}(k, s)$ and $Z_{\mathfrak{a} \mathfrak{b}}(k, 0, s)$ are analytic at $s=1$. Also $\phi_{\mathfrak{a} \mathfrak{b}}(s)$ has a simple pole at $s=1$ with the above residue.

The expansion for $E_{\mathfrak{a}}(z, s, \Lambda)$ is very similar to equations (3.4) - (3.6), but only for $\Lambda$ in $\mathcal{V}_{\mathfrak{a}} \cap \mathcal{V}_{\mathfrak{b}}$ do we have $E_{\mathfrak{a}}\left(\sigma_{\mathfrak{b}}(z+1), s, \Lambda\right)=E_{\mathfrak{a}}\left(\sigma_{\mathfrak{b}}(z), s, \Lambda\right)$. In that case

$$
E_{\mathfrak{a}}\left(\sigma_{\mathfrak{b}} z, s, \Lambda\right)=\phi_{\mathfrak{a} \mathfrak{b}}(s, \Lambda) y^{1-s}+\sum_{k \neq 0} \phi_{\mathfrak{a} \mathfrak{b}}(k, s, \Lambda) W_{s}(k z),
$$

for $\operatorname{Re}(s)>R_{\Lambda}+1$. Also

$$
\begin{aligned}
\phi_{\mathfrak{a} \mathfrak{b}}(s, \Lambda) & =\sqrt{\pi} \Gamma(s)^{-1} \Gamma\left(s-\frac{1}{2}\right) Z_{\mathfrak{a} \mathfrak{b}}(0,0, s, \Lambda), \\
\phi_{\mathfrak{a} \mathfrak{b}}(k, s, \Lambda) & =\pi^{s} \Gamma(s)^{-1}|k|^{s-1} Z_{\mathfrak{a} \mathfrak{b}}(k, 0, s, \Lambda) .
\end{aligned}
$$

We shall extend the domain of $E_{\mathfrak{a}}(z, s, \Lambda)$ in section 6 by analytically continuing these Fourier coefficients. 


\section{Constructing holomorphic Eisenstein series of weight 2.}

In this section we construct the basis mentioned before proposition 2.1. The Eisenstein series of even weight $k \geq 4$ is given by the absolutely convergent series:

$$
E_{\mathfrak{a}, k}(z)=\sum_{\gamma \in \Gamma_{\mathfrak{a}} \backslash \Gamma} j\left(\sigma_{\mathfrak{a}}^{-1} \gamma, z\right)^{-k} .
$$

For $k=2$ the above convergence is only conditional. Given our knowledge of $E_{\mathfrak{a}}(z, s)$ we may construct Eisenstein series of weight 2 in a straightforward manner. Set

$$
E_{\mathfrak{a}, 2}(z)=2 i \lim _{s \rightarrow 1} \frac{d}{d z} E_{\mathfrak{a}}(z, s),
$$

where $\frac{d}{d z}=\frac{1}{2}\left(\frac{d}{d x}-i \frac{d}{d y}\right)$. This will clearly have weight 2 since $\frac{d}{d z} \gamma z=j(\gamma, z)^{-2}$. At the cusp $\mathfrak{b}$

$$
j\left(\sigma_{\mathfrak{b}}, z\right)^{-2} E_{\mathfrak{a}, 2}\left(\sigma_{\mathfrak{b}} z\right)=2 i \lim _{s \rightarrow 1} \frac{d}{d z} E_{\mathfrak{a}}\left(\sigma_{\mathfrak{b}} z, s\right) .
$$

Recall equation (3.4). Differentiating the constant term of $E_{\mathfrak{a}}$ we get

$$
\delta_{\mathfrak{a} \mathfrak{b}} s y^{s-1}+(1-s) \phi_{\mathfrak{a} \mathfrak{b}}(s) y^{-s}
$$

which has limit $\delta_{\mathfrak{a} \mathfrak{b}}-\frac{1}{y} \operatorname{Vol}(\Gamma \backslash \mathfrak{H})^{-1}$ as $s \rightarrow 1$.

For the other terms, with $n \neq 0$,

$$
\begin{aligned}
2 i \lim _{s \rightarrow 1} \frac{d}{d z} \phi_{\mathfrak{a} \mathfrak{b}}(n, s) W_{s}(n z) & =2 i \frac{d}{d z}\left(\lim _{s \rightarrow 1} \phi_{\mathfrak{a} \mathfrak{b}}(n, s) W_{s}(n z)\right) \\
& =2 i \frac{d}{d z}\left(\phi_{\mathfrak{a} \mathfrak{b}}(n, 1) W_{1}(n z)\right) \\
& =2 i \phi_{\mathfrak{a} \mathfrak{b}}(n, 1) \frac{d}{d z} e^{2 \pi i n x-2 \pi|n| y}
\end{aligned}
$$

and this derivative is 0 if $n<0$ and $-4 \pi n e^{2 \pi i n z}$ if $n>0$. Putting these results together

$$
j\left(\sigma_{\mathfrak{b}}, z\right)^{-2} E_{\mathfrak{a}, 2}\left(\sigma_{\mathfrak{b}} z\right)=\delta_{\mathfrak{a} \mathfrak{b}}-\frac{1}{y} \operatorname{Vol}(\Gamma \backslash \mathfrak{H})^{-1}-4 \pi^{2} \sum_{n=1}^{\infty} n Z_{\mathfrak{a} \mathfrak{b}}(n, 0,1) e^{2 \pi i n z}
$$

Any difference $E_{\mathfrak{a}, 2}(z)-E_{\mathfrak{b}, 2}(z)$ will be holomorphic with constant term $\delta_{\mathfrak{a} \mathfrak{c}}-\delta_{\mathfrak{b c}}$ at the cusp $\mathfrak{c}$. If $\Gamma$ has $m$ cusps then $m-1$ of these differences will form the basis for $\mathcal{E}_{2}(\Gamma)$ required in proposition 2.1. Also, note that any bound

$$
Z_{\mathfrak{a} \mathfrak{b}}(n, 0,1) \ll n^{1-\epsilon}
$$

for $\epsilon>0$ would improve proposition 2.3. 


\section{The residue of $E_{\mathfrak{a}}(z, s, \Lambda)$ at $s=1$.}

Assume for the moment that $E_{\mathfrak{a}}(z, s, \Lambda)$ has a meromorphic continuation to $\operatorname{Re}(s)>1-\varepsilon$ for some $\varepsilon>0$. We will see that this can be justified for $\Lambda \in \mathcal{W}(\Gamma)$. At $s=1$ we necessarily have a pole, see remark 5.6 below. Let $R_{\mathfrak{a}}(z, \Lambda)=\operatorname{Res}_{s=1} E_{\mathfrak{a}}(z, s, \Lambda)$. From (1.4) we have $R_{\mathfrak{a}}(\gamma z, \Lambda)=R_{\mathfrak{a}}(z, \Lambda)+\operatorname{Vol}(\Gamma \backslash \mathfrak{H})^{-1} \Lambda\left(\gamma^{-1}\right)$ for all $\gamma \in \Gamma$ and all $z \in \mathfrak{H}$. Also $R_{\mathfrak{a}}(z, \Lambda)$ is harmonic with $\Delta R_{\mathfrak{a}}(z, \Lambda)=0$. Now $\operatorname{Vol}(\Gamma \backslash \mathfrak{H})^{-1}\left(-P_{\mathfrak{a}}(z, \Lambda)-\overline{Q_{\mathfrak{a}}(z, \Lambda)}\right)$ has the same properties so that $P_{\mathfrak{a}}+\overline{Q_{\mathfrak{a}}}+R_{\mathfrak{a}}$ is automorphic and harmonic.

To show that $P_{\mathfrak{a}}(z, \Lambda)+\overline{Q_{\mathfrak{a}}(z, \Lambda)}$ is bounded for $z \in \mathfrak{F}$ is not hard. Each of $P_{\mathfrak{a}}$ and $Q_{\mathfrak{a}}$ are linear combinations of integrals of cusp forms, which are certainly bounded, and terms of the form $h(z)=\operatorname{Re} \int_{\mathfrak{a}}^{z} g(w) d w$ for $g \in \mathcal{M}_{2}(\gamma)$ with Fourier expansions as in (2.1). Thus

$$
\begin{aligned}
h\left(\sigma_{\mathfrak{b}} z\right) & =\operatorname{Re} \int_{\mathfrak{a}}^{\sigma_{\mathfrak{b}}(i)} g(w) d w+\operatorname{Re} \int_{\sigma_{\mathfrak{b}}(i)}^{\sigma_{\mathfrak{b}}(z)} g(w) d w \\
& =M_{\mathfrak{a}}+\operatorname{Re} \int_{i}^{z} j\left(\sigma_{\mathfrak{b}}, w\right)^{-2} g\left(\sigma_{\mathfrak{b}} w\right) d w \\
& =M_{\mathfrak{a}}+\operatorname{Re} \int_{i}^{z} \sum_{n=0}^{\infty} c_{\mathfrak{b}}(n) e^{2 \pi i n w} d w \\
& =N_{\mathfrak{a}}+c_{\mathfrak{b}}(0) x+\operatorname{Re} \sum_{n=1}^{\infty} \frac{c_{\mathfrak{b}}(n)}{2 \pi i n} e^{2 \pi i n z}
\end{aligned}
$$

where $M_{\mathfrak{a}}, N_{\mathfrak{a}}$ are constants. The last line follows since $c_{\mathfrak{b}}(0) \in \mathbb{R}$. Therefore $h\left(\sigma_{\mathfrak{b}} z\right)$ is bounded as $y \rightarrow \infty$. This shows that $P_{\mathfrak{a}}+\overline{Q_{\mathfrak{a}}}$ is bounded on $\mathfrak{F}$. Note that the only functions that are automorphic, harmonic and bounded are the constant functions. We have proved the following.

Proposition 5.1. If $E_{\mathfrak{a}}(z, s, \Lambda)$ is meromorphic at $s=1$ then it has a pole there and

$$
\operatorname{Res}_{s=1} E_{\mathfrak{a}}(z, s, \Lambda)=-\operatorname{Vol}(\Gamma \backslash \mathfrak{H})^{-1}\left(P_{\mathfrak{a}}(z, \Lambda)+\overline{Q_{\mathfrak{a}}(z, \Lambda)}+U_{\mathfrak{a}}(z, \Lambda)\right)
$$

for $P_{\mathfrak{a}}, Q_{\mathfrak{a}}$ as defined in $(2.2),(2.3)$ and $U_{\mathfrak{a}}(z, \Lambda)$ an automorphic and harmonic function. Furthermore, $U_{\mathfrak{a}}(z, \Lambda)$ is constant if the residue $R_{\mathfrak{a}}$ is bounded on $\mathfrak{F}$.

When $\Lambda \in \mathcal{W}(\Gamma)$ we can find the residue exactly. In [O'S1] the case $\Lambda=\Lambda_{f}$ for $f \in \mathcal{S}_{2}(\Gamma)$ is treated. There it is shown that $E_{\mathfrak{a}}(z, s, \Lambda)$ has a meromorphic continuation to all $s \in \mathbb{C}$ with Fourier expansion (3.7) at each cusp. At $s=1$ the Fourier expansion of the residue shows that it is bounded on $\mathfrak{F}$ (see also [O'S2]). For $\overline{\Lambda_{f}}$ the same theory applies since we may consider $\overline{E_{\mathfrak{a}}}(z, \bar{s}, \Lambda)$. We obtain:

Proposition 5.2. For $\Lambda \in \mathcal{W}(\Gamma)$ the Eisenstein series $E_{\mathfrak{a}}(z, s, \Lambda)$ has a meromorphic continuation to all $s \in \mathbb{C}$. At $s=1$ it has a simple pole with a residue that is bounded on $\mathfrak{F}$.

It follows that $U_{\mathfrak{a}}(z, \Lambda)$ is constant. By letting $z \rightarrow \mathfrak{a}$ we can show that it is actually zero with the following lemma. 
Lemma 5.3. We have $\phi_{\mathfrak{a} \mathfrak{a}}(s, \Lambda)=0$.

Proof: The Kloosterman sum $S_{\mathfrak{a} \mathfrak{a}}(0,0, c, \Lambda)$ is zero since if $c \in C_{\mathfrak{a} a}, \gamma \in \sigma_{\mathfrak{a}}{ }^{-1} \Gamma \sigma_{\mathfrak{a}}$ and $\gamma_{c}=c$ then $\gamma^{-1} \in \sigma_{\mathfrak{a}}{ }^{-1} \Gamma \sigma_{\mathfrak{a}}$ and $\left(\gamma^{-1}\right)_{c}=c$ also and they both contribute a total of 0 to the sum. Similarly if $\gamma$ is its own inverse. Hence, recalling section 3 , we have $\phi_{\mathfrak{a} \mathfrak{a}}(s, \Lambda)=0$ for $\operatorname{Re}(s)>3$ say and the lemma follows by analytic continuation.

We have proved the following.

Proposition 5.4. For $\Lambda \in \mathcal{W}(\Gamma)$ the Eisenstein series $E_{\mathfrak{a}}(z, s, \Lambda)$ has a simple pole at $s=1$ and $($ see $(2.2),(2.3))$,

$$
\operatorname{Res}_{s=1} E_{\mathfrak{a}}(z, s, \Lambda)=-\operatorname{Vol}(\Gamma \backslash \mathfrak{H})^{-1}\left(P_{\mathfrak{a}}(z, \Lambda)+\overline{Q_{\mathfrak{a}}(z, \Lambda)}\right) .
$$

Note also that $E_{\mathfrak{a}}(z, s, \Lambda)$ has a functional equation similar to that of theorem 4.1 in [O'S1]. For $\Lambda \notin \mathcal{W}(\Gamma)$ complications arise because we cannot take advantage of a simple Fourier expansion at each cusp.

Remark 5.5. Lemma 5.3 gives the entries on the diagonal of the 'scattering matrix' $\phi_{\mathfrak{a} \mathfrak{b}}(s, \Lambda)$. In [Ch-O'S] it is shown that

$$
\phi_{\mathfrak{a} \mathfrak{b}}\left(s, \Lambda_{f}\right)=-\phi_{\mathfrak{a} \mathfrak{b}}(s) \int_{\mathfrak{a}}^{\mathfrak{b}} f(w) d w-\frac{2 i s}{2 s-1} \int_{\Gamma \backslash \mathfrak{H}} y^{k / 2} f(z) E_{-k, \mathfrak{a}}(z, s) E_{\mathfrak{b}}(z, s) \frac{d x d y}{y^{2}}
$$

for

$$
E_{-k, \mathfrak{a}}(z, s)=\sum_{\gamma \in \Gamma_{\mathfrak{a}} \backslash \Gamma} \operatorname{Im}\left(\sigma_{\mathfrak{a}}^{-1} \gamma z\right)^{s}\left(\frac{j\left(\sigma_{\mathfrak{a}}^{-1} \gamma, z\right)}{\left|j\left(\sigma_{\mathfrak{a}}-1 \gamma, z\right)\right|}\right)^{k}
$$

see (3.5) and (3.8). This corrects an earlier formula in [Ch-Go]. How does this generalize to all $\Lambda \in \mathcal{V}_{\mathfrak{a}} \cap \mathcal{V}_{\mathfrak{b}}$ ?

Remark 5.6. If $E_{\mathfrak{a}}(z, s, \Lambda)$ with $\Lambda \in \mathcal{W}(\Gamma)$ has a pole of order $n>1$ at $s=1$ then

$$
E_{\mathfrak{a}}(z, s, \Lambda)=A_{-n}(z, \Lambda)(s-1)^{-n}+A_{-n+1}(z, \Lambda)(s-1)^{-n+1}+\ldots
$$

and $A_{-n}(z, \Lambda)$ is automorphic, harmonic and, by examining its Fourier expansion, bounded. It is, therefore, constant, and by letting $z \rightarrow \mathfrak{a}$ as before, this constant must be zero. This shows that the pole at $s=1$ must be simple. Since $E_{\mathfrak{a}}(z, s)$ has no poles in $\operatorname{Re}(s)>1 / 2$, except perhaps in $(1 / 2,1]$, a similar argument to the above shows that $E_{\mathfrak{a}}(z, s, \Lambda)$ is also analytic for all values of $s$ in $\operatorname{Re}(s)>1 / 2$ except for possible poles in $(1 / 2,1]$.

\section{Analytic continuation using Selberg-Kloosterman zeta functions.}

Theorem 2.2 may be improved by using results for the Selberg-Kloosterman zeta function that was defined in section 3 . 
Proposition 6.1. For $\Lambda \in \mathcal{V}_{\mathfrak{a}}(\Gamma), P_{\mathfrak{a}}, Q_{\mathfrak{a}}$ as in (2.2), (2.3) and $R_{\Lambda}$ as defined in (2.4) the Fourier coefficients of the series $E_{\mathfrak{a}}(z, s, \Lambda)$ have analytic continuations to $\operatorname{Re}(s)>R_{\Lambda} / 2+1$. For congruence groups this can be improved to $\operatorname{Re}(s)>R_{\Lambda} / 2+3 / 4$.

PROOF: Recall the notation from the beginning of section 3. For $\gamma$ in $\Gamma$ with $c=\gamma_{c}>0$ and $z=\frac{-\gamma_{d}}{c}+\frac{i}{c}$ we have $\gamma z=\frac{\gamma_{a}}{c}+\frac{i}{c}$. Therefore,

$$
\begin{aligned}
2 \pi i \Lambda\left(\sigma_{\mathfrak{a}} \gamma \sigma_{\mathfrak{a}}{ }^{-1}\right)= & 2 \pi i\left(P_{\mathfrak{a}}\left(\sigma_{\mathfrak{a}} \gamma z\right)-P_{\mathfrak{a}}\left(\sigma_{\mathfrak{a}} z\right)+\overline{Q_{\mathfrak{a}}\left(\sigma_{\mathfrak{a}} \gamma z\right)-Q_{\mathfrak{a}}\left(\sigma_{\mathfrak{a}} z\right)}\right) \\
= & \sum_{k>0} \frac{p_{\mathfrak{a}}(k)}{k}\left(e^{2 \pi i k\left(\frac{\gamma_{\mathfrak{a}}}{c}+\frac{i}{c}\right)}-e^{2 \pi i k\left(\frac{-\gamma_{d}}{c}+\frac{i}{c}\right)}\right) \\
& \quad+\sum_{k>0} \frac{\overline{q_{\mathfrak{a}}(k)}}{k}\left(e^{-2 \pi i k \overline{\left(\frac{\gamma_{a}}{c}+\frac{i}{c}\right)}}-e^{-2 \pi i k \overline{\left(\frac{-\gamma_{d}}{c}+\frac{i}{c}\right)}}\right) \\
= & \sum_{k \neq 0} \frac{p_{\mathfrak{a}}(k)}{|k|} e^{\frac{-2 \pi|k|}{c}}\left(e^{-2 \pi i \frac{k \gamma_{d}}{c}}-e^{2 \pi i \frac{k \gamma_{a}}{c}}\right),
\end{aligned}
$$

where, for convenience, we define $p_{\mathfrak{a}}(-k)=\overline{q_{\mathfrak{a}}(k)}$ for $-k<0$. Then, from (3.2) we have

$$
\begin{aligned}
2 \pi i S_{\mathfrak{a} a}(m, n, c, \Lambda) & =\sum_{\gamma} 2 \pi i \Lambda\left(\sigma_{\mathfrak{a}} \gamma \sigma_{\mathfrak{a}}{ }^{-1}\right) e^{2 \pi i\left(n \frac{\gamma_{a}}{c}+m \frac{\gamma_{d}}{c}\right)} \\
& =\sum_{k \neq 0} \frac{p_{\mathfrak{a}}(k)}{|k|} e^{\frac{-2 \pi|k|}{c}} \sum_{\gamma}\left(e^{2 \pi i \frac{-k \gamma_{d}+n \gamma_{a}+m \gamma_{d}}{c}}-e^{2 \pi i \frac{k \gamma_{a}+n \gamma_{a}+m \gamma_{d}}{c}}\right) \\
& =\sum_{k \neq 0} \frac{p_{\mathfrak{a}}(k)}{|k|} e^{\frac{-2 \pi|k|}{c}}\left(S_{\mathfrak{a} \mathfrak{a}}(m-k, n, c)-S_{\mathfrak{a} \mathfrak{a}}(m, n+k, c)\right) .
\end{aligned}
$$

Use the representation

$$
\frac{1}{2 \pi i} \int_{(u)} \Gamma(w)\left(\frac{2 \pi k}{c}\right)^{-w} d w=e^{\frac{-2 \pi k}{c}},
$$

where the integral is from $u-i \infty$ to $u+i \infty$ with $u>0$, to write

$$
\begin{gathered}
Z_{\mathfrak{a} \mathfrak{a}}(m, n, s, \Lambda)=\sum_{k \neq 0} \frac{p_{\mathfrak{a}}(k)}{|k|} \sum_{c \in C_{\mathfrak{a} \mathfrak{a}}} e^{\frac{-2 \pi|k|}{c}}\left(\frac{S_{\mathfrak{a} \mathfrak{a}}(m-k, n, c)}{c^{2 s}}-\frac{S_{\mathfrak{a} \mathfrak{a}}(m, n+k, c)}{c^{2 s}}\right) \\
=\frac{1}{2 \pi i} \int_{(u)} \sum_{k \neq 0} \frac{p_{\mathfrak{a}}(k)}{|k|}(2 \pi k)^{-w} \Gamma(w) \sum_{c \in C_{\mathfrak{a} a}}\left(\frac{S_{\mathfrak{a} \mathfrak{a}}(m-k, n, c)}{c^{2 s-w}}-\frac{S_{\mathfrak{a} \mathfrak{a}}(m, n+k, c)}{c^{2 s-w}}\right) d w \\
=\frac{1}{2 \pi i} \int_{(u)} \frac{\Gamma(w)}{(2 \pi)^{w}} \sum_{k \neq 0} \frac{p_{\mathfrak{a}}(k)}{|k| k^{w}}\left(Z_{\mathfrak{a} \mathfrak{a}}\left(m-k, n, s-\frac{w}{2}\right)-Z_{\mathfrak{a} \mathfrak{a}}\left(m, n+k, s-\frac{w}{2}\right)\right) d w .
\end{gathered}
$$

Now for $\Gamma$ an arbitrary Fuchsian group of the first kind we have by elementary estimates that $Z_{\mathfrak{a} \mathfrak{b}}(m, n, s)$ is absolutely convergent for $\operatorname{Re}(s)>1$, see [Iw2]. In fact $Z_{\mathfrak{a} \mathfrak{b}}(m, n, s)=O(1)$ for $\operatorname{Re}(s) \geq 1+\varepsilon$ where the implied constant depends only on $\Gamma$ and $\varepsilon>0$. Therefore,

$$
Z_{\mathfrak{a} \mathfrak{a}}(m, n, s, \Lambda) \ll \int_{(u)} \frac{|\Gamma(w)|}{(2 \pi)^{u}} \sum_{k \neq 0} \frac{1}{|k|^{u+1-R_{\Lambda}}} d w
$$


for $\operatorname{Re}(s)-u / 2 \geq 1+\varepsilon$ and $u+1-R_{\Lambda}>1$. In other words $Z_{\mathfrak{a} \mathfrak{a}}(m, n, s, \Lambda)=O(1)$ for $\operatorname{Re}(s) \geq R_{\Lambda} / 2+1+\varepsilon$ with the implied constant depending only on $\Gamma$ and $\varepsilon>0$ as before.

For congruence groups we have that $S_{\mathfrak{a} \mathfrak{b}}(m, n, c) \ll c^{1 / 2+\varepsilon}$ by Weil's bound. Therefore, $Z_{\mathfrak{a} \mathfrak{b}}(m, n, s)=O(1)$ for $\operatorname{Re}(s) \geq 3 / 4+\varepsilon$. This gives the improvement $Z_{\mathfrak{a} \mathfrak{b}}(m, n, s, \Lambda)=O(1)$ for $\operatorname{Re}(s) \geq 3 / 4+R_{\Lambda} / 2+\varepsilon$.

These results show that the Fourier coefficients $\phi_{\mathfrak{a} \mathfrak{a}}(s, \Lambda), \phi_{\mathfrak{a} \mathfrak{a}}(k, s, \Lambda)$ of $E_{\mathfrak{a}}\left(\sigma_{\mathfrak{a}} z, s, \Lambda\right)$, given by (3.8), (3.9), are analytic functions for $\operatorname{Re}(s)$ in the above ranges.

These estimates may also be used to show that $E_{\mathfrak{a}}(z, s, \Lambda)$ is of slow growth for $s$ in vertical strips.

Proposition 6.2. Let

$$
X(z, s)=\sum_{k \neq 0} \frac{|k|^{s-1}}{\Gamma(s)} x_{k}(s) W_{s}(k z)
$$

Suppose $s=\sigma+i t$ is in the half plane $1 / 2<\sigma_{0} \leq \sigma$ and $y>0$. If $\left|x_{k}(s)\right| \leq C$ for this $s$ and for all $k$ then we have

$$
X(z, s) \ll y^{-\sigma}\left(1+y^{-\sigma-1 / 2}\right)^{\varepsilon}(1+|t|)^{\varepsilon}
$$

with the implied constant depending on $\sigma_{0}, C$ and $\varepsilon>0$.

Proof: The Bessel $K$-function $K_{w}(y)$ for $y>0$ and $w \in \mathbb{C}$ has the following integral representation

$$
K_{w}(y)=\frac{1}{2} \int_{0}^{\infty} e^{\frac{-y}{2}\left(t+\frac{1}{t}\right)} t^{w-1} d t
$$

and it can be seen to be an entire function of $w$. Using (6.1) we can show that for $w=u+i v$ and $u \geq 0$ we have

$$
\left|K_{w}(y)\right| \leq \Gamma(u+2)\left(2+y^{-u-1}\right) e^{-y} .
$$

For $\operatorname{Re}(w)>-1 / 2$ we also have the expression

$$
K_{w}(y)=\frac{1}{\sqrt{\pi}} \Gamma(w+1 / 2)\left(\frac{2}{y}\right)^{w} \int_{0}^{\infty} \frac{\cos (t y)}{(t+1)^{w+1 / 2}} d t .
$$

Consequently, we obtain

$$
\begin{aligned}
\left|K_{w}(y)\right| & \leq \frac{1}{\sqrt{\pi}}|\Gamma(w+1 / 2)|\left(\frac{2}{y}\right)^{u} \int_{0}^{\infty} \frac{1}{(t+1)^{u+1 / 2}} d t \\
& =\frac{1}{2}|\Gamma(w+1 / 2)|\left(\frac{2}{y}\right)^{u} \frac{\Gamma(u)}{\Gamma(u+1 / 2)} .
\end{aligned}
$$

Therefore, for $y>0,0<\varepsilon \leq \operatorname{Re}(s)$ and $s=\sigma+i t$ we have the two estimates

$$
\begin{aligned}
& K_{s-1 / 2}(2 \pi|k| y) \ll\left(2+(2 \pi|k| y)^{-\sigma-1 / 2}\right) e^{-2 \pi|k| y} \\
& K_{s-1 / 2}(2 \pi|k| y) \ll|\Gamma(s)||k|^{1 / 2-\sigma} y^{1 / 2-\sigma}
\end{aligned}
$$


the implied constants depending on $\varepsilon$ and $\sigma$. The first has exponential decay as $y \rightarrow \infty$ and the second has exponential decay as $|t| \rightarrow \infty$. We employ a combination of these bounds depending on the parameter $0 \leq r \leq 1$. We have

$$
\begin{aligned}
\frac{|k|^{s-1}}{\Gamma(s)} x_{k}(s) W_{s}(k z) \ll & \frac{|k|^{\sigma-1}}{|\Gamma(s)|} \sqrt{|k| y}\left[|\Gamma(s)||k|^{1 / 2-\sigma} y^{1 / 2-\sigma}\right]^{1-r} \\
\times & {\left[\left(2+(2 \pi|k| y)^{-\sigma-1 / 2}\right) e^{-2 \pi|k| y}\right]^{r} } \\
= & y^{1-\sigma-r / 2+r \sigma}|\Gamma(s)|^{-r}|k|^{-r / 2+r \sigma}\left(2+(2 \pi|k| y)^{-\sigma-1 / 2}\right)^{r} e^{-2 \pi|k| y r} .
\end{aligned}
$$

Note that $2+(2 \pi|k| y)^{-\sigma-1 / 2} \leq 2+y^{-\sigma-1 / 2}$ and $\sum_{k \neq 0}|k|^{u} e^{-v|k|} \ll v^{-u-1} \Gamma(u+1)$ for $u, v>0$ so that

$\sum_{k \neq 0}\left(2+(2 \pi|k| y)^{-\sigma-1 / 2}\right)^{r}|k|^{-r / 2+r \sigma} e^{-2 \pi|k| y} \ll\left(1+y^{-\sigma-1 / 2}\right)^{r}(2 \pi y r)^{r / 2-1-r \sigma} \Gamma(1-r / 2+r \sigma)$,

for $0<r \leq 1$. This means that

$$
X(z, s) \ll(2 \pi)^{-r \sigma-1} \Gamma(r(\sigma-1 / 2)+1) y^{-\sigma}\left(1+y^{-\sigma-1 / 2}\right)^{r}|t|^{-r(\sigma-1 / 2)} e^{\frac{\pi|t| r}{2}} .
$$

Now for $|t| \leq e$ choose $r=\varepsilon$. It follows that

$$
X(z, s) \ll y^{-\sigma}\left(1+y^{-\sigma-1 / 2}\right)^{\varepsilon} .
$$

For $|t|>e$ set $r=\frac{\varepsilon \ln |t|}{|t|} \leq \varepsilon$ and note that $|t|^{r} \leq e$ and $e^{\frac{\pi|t| r}{2}}=e^{\frac{\varepsilon \pi}{2}}$ implying

$$
X(z, s) \ll y^{-\sigma}\left(1+y^{-\sigma-1 / 2}\right)^{\varepsilon}|t|^{\varepsilon} .
$$

By (3.7), (3.8), (3.9) we have

$$
\begin{aligned}
E_{\mathfrak{a}}\left(\sigma_{\mathfrak{a}} z, s, \Lambda\right)= & \sqrt{\pi} \frac{\Gamma(s-1 / 2)}{\Gamma(s)} Z_{\mathfrak{a} \mathfrak{a}}(0,0, s, \Lambda) y^{1-s} \\
& +\sum_{k \neq 0} \frac{|k|^{s-1}}{\Gamma(s)} \pi^{s} Z_{\mathfrak{a} \mathfrak{a}}(k, 0, s, \Lambda) W_{s}(k z) .
\end{aligned}
$$

Proposition 6.1 demonstrated that $Z_{\mathfrak{a} a}(m, n, s, \Lambda)=O(1)$ when $\operatorname{Re}(s) \geq R$ for a particular constant $R$ depending on $\Gamma$. Thus

$$
\begin{aligned}
E_{\mathfrak{a}}\left(\sigma_{\mathfrak{a}} z, s, \Lambda\right) & \ll \frac{|\Gamma(s-1 / 2)|}{|\Gamma(s)|} y^{1-\sigma}+X(z, s) \\
& \ll \frac{1}{\sqrt{1+|t|}} y^{1-\sigma}+y^{-\sigma}\left(1+y^{-\sigma-1 / 2}\right)^{\varepsilon}(1+|t|)^{\varepsilon} .
\end{aligned}
$$

Putting all this together we obtain: 
Theorem 6.3. For $\Lambda \in \mathcal{V}_{\mathfrak{a}}(\Gamma)$ the Eisenstein series $E_{\mathfrak{a}}(z, s, \Lambda)$ has an analytic continuation to $\operatorname{Re}(s) \geq R$ and in this domain we have

$$
E_{\mathfrak{a}}\left(\sigma_{\mathfrak{a}} z, s, \Lambda\right) \ll y^{1-\sigma}+y^{-\sigma}\left(1+y^{-\sigma-1 / 2}\right)^{\varepsilon}(1+|t|)^{\varepsilon}
$$

with $s=\sigma+i t$ and $\varepsilon>0$. The implied constant depends only on $R$ and $\varepsilon$, for $R$ any constant satisfying

$$
R>\left\{\begin{aligned}
R_{\Lambda} / 2+3 / 4 & \text { for } \Gamma \text { a congruence group } \\
R_{\Lambda} / 2+1 & \text { for general } \Gamma
\end{aligned}\right.
$$

where $R_{\Lambda}$ is defined in (2.4).

To make things clearer we combine (6.6) with proposition 2.3 and get, for $\Gamma_{1}$ a congruence group and $\Gamma_{2}$ an arbitrary Fuchsian group of the first kind

$$
R>\left\{\begin{array}{rl}
1 & \text { if } \Lambda \in \mathcal{W}\left(\Gamma_{1}\right) \\
5 / 4 & \text { if } \Lambda \in \mathcal{V}\left(\Gamma_{1}\right)
\end{array}, R>\left\{\begin{aligned}
3 / 2 & \text { if } \Lambda \in \mathcal{W}\left(\Gamma_{2}\right) \\
2 & \text { if } \Lambda \in \mathcal{V}\left(\Gamma_{2}\right)
\end{aligned}\right.\right.
$$

\section{Estimating additive character sums.}

From theorem 6.3 we know that $E_{\mathfrak{a}}(z, s, \Lambda) \ll|t|^{\varepsilon}$ as $t=\operatorname{Im}(s) \rightarrow \pm \infty$ for $\operatorname{Re}(s) \geq R$ as in (6.7), the implied constant depending on $z$ and $R$. Thus

$$
I(z, R, T)=\frac{1}{2 \pi i} \int_{R-i \infty}^{R+i \infty} E_{\mathfrak{a}}(z, s, \Lambda) \frac{T^{s}}{\operatorname{Im}\left(\sigma_{\mathfrak{a}}^{-1} z\right)^{s}} \frac{1}{s(s+1)} d s
$$

is absolutely convergent. Now $E_{\mathfrak{a}}(z, s, \Lambda)$ as defined in (1.2) is absolutely convergent for $\operatorname{Re}(s)>R_{0}=R_{\Lambda}+1$ by theorem 2.2 . We have

$$
\begin{aligned}
\frac{1}{2 \pi i} \int_{R_{0}-i \infty}^{R_{0}+i \infty} \operatorname{Im}\left(\sigma_{\mathfrak{a}}{ }^{-1} \gamma z\right)^{s} & \frac{T^{s}}{\operatorname{Im}\left(\sigma_{\mathfrak{a}}{ }^{-1} z\right)^{s}} \frac{1}{s(s+1)} d s \\
& =\frac{1}{2 \pi i} \int_{R_{0}-i \infty}^{R_{0}+i \infty} \frac{T^{s}}{\left|j\left(\sigma_{\mathfrak{a}}{ }^{-1} \gamma \sigma_{\mathfrak{a}}, \sigma_{\mathfrak{a}}{ }^{-1} z\right)\right|^{2 s}} \frac{1}{s(s+1)} d s \\
& =1-\frac{\left|j\left(\sigma_{\mathfrak{a}}{ }^{-1} \gamma \sigma_{\mathfrak{a}}, \sigma_{\mathfrak{a}}{ }^{-1} z\right)\right|^{2}}{T} \text { or } 0,
\end{aligned}
$$

depending on whether $T>\left|j\left({\sigma_{\mathfrak{a}}}^{-1} \gamma \sigma_{\mathfrak{a}},{\sigma_{\mathfrak{a}}}^{-1} z\right)\right|^{2}$ or not. Set

$$
\mathcal{J}_{\mathfrak{a}}(z, T, \Lambda)=\sum_{\substack{\gamma \in \Gamma_{\mathfrak{a}} \backslash \Gamma \\\left|j\left(\sigma_{\mathfrak{a}}{ }^{-1} \gamma \sigma_{\mathfrak{a}}, \sigma_{\mathfrak{a}}{ }^{-1} z\right)\right|^{2}<T}} \Lambda(\gamma)\left(1-\frac{\left|j\left(\sigma_{\mathfrak{a}}{ }^{-1} \gamma \sigma_{\mathfrak{a}}, \sigma_{\mathfrak{a}}{ }^{-1} z\right)\right|^{2}}{T}\right)
$$

the sum we are trying to estimate. Then $\mathcal{J}_{\mathfrak{a}}(z, T, \Lambda)=I\left(z, R_{0}, T\right)$. Since $I(z, R, T)=$ $I\left(z, R_{0}, T\right)$ by moving the line of integration, (see remark 5.6), we obtain

$$
\mathcal{J}_{\mathfrak{a}}\left(\sigma_{\mathfrak{a}} z, T, \Lambda\right)=I\left(\sigma_{\mathfrak{a}} z, R, T\right) \ll\left(y^{1-2 R}+y^{-3 R}\right) T^{R}
$$

by substituting (6.5) into (7.1). So we have proved 
Theorem 7.1. For $\Lambda \in \mathcal{V}_{\mathfrak{a}}(\Gamma)$ and $\mathcal{J}_{\mathfrak{a}}(z, T, \Lambda)$ as defined in (7.2) we have

$$
\mathcal{J}_{\mathfrak{a}}\left(\sigma_{\mathfrak{a}} z, T, \Lambda\right) \ll\left(y^{1-2 R}+y^{-3 R}\right) T^{R}
$$

for $R$ as in (6.7).

Thus, for example, if $\Gamma$ is a congruence group we may take $R=5 / 4+\varepsilon$ and in that case

$$
\mathcal{J}_{\mathfrak{a}}\left(\sigma_{\mathfrak{a}} z, T, \Lambda\right) \ll\left(y^{-3 / 2-\varepsilon}+y^{-15 / 4-\varepsilon}\right) T^{5 / 4+\varepsilon}
$$

This is improved to

$$
\mathcal{J}_{\mathfrak{a}}\left(\sigma_{\mathfrak{a}} z, T, \Lambda\right) \ll\left(y^{-1-\varepsilon}+y^{-3-\varepsilon}\right) T^{1+\varepsilon},
$$

for $\Lambda \in \mathcal{W}(\Gamma)$ where the implied constants in (7.3), (7.4) depend only on $\varepsilon$ and $\Lambda$.

With conjecture 1.1 we can move the line of integration to $\operatorname{Re}(s)=1-\epsilon$ and get

$$
\mathcal{J}_{\mathfrak{a}}(z, T, \Lambda)=R_{\mathfrak{a}}(z, \Lambda) \frac{T}{2 \operatorname{Im}\left(\sigma_{\mathfrak{a}}^{-1} z\right)}+I(z, 1-\epsilon, T)
$$

This gives

Theorem 7.2. If we assume conjecture 1.1 then

$$
\mathcal{J}_{\mathfrak{a}}(z, T, \Lambda)=\frac{-T}{2 \operatorname{Im}\left(\sigma_{\mathfrak{a}}^{-1} z\right) \operatorname{Vol}(\Gamma \backslash \mathfrak{H})}\left(P_{\mathfrak{a}}(z, \Lambda)+\overline{Q_{\mathfrak{a}}(z, \Lambda)}+U_{\mathfrak{a}}(z, \Lambda)\right)+O\left(T^{1-\epsilon}\right)
$$

as $T \rightarrow \infty$ where the implied constant depends on $z, \Lambda$ and $\Gamma$. The functions $P_{\mathfrak{a}}, Q_{\mathfrak{a}}$ and $U_{\mathfrak{a}}$ are as in proposition 5.1 .

If $\Lambda$ is cuspidal and $\Gamma$ is a congruence subgroup then we may use Petridis' bound [Pe] to obtain a much stronger unconditional result. ${ }^{3}$

Theorem 7.3. For $\Lambda$ cuspidal and $\Gamma$ a congruence subgroup

$$
\sum_{\substack{\gamma \in \Gamma_{\mathfrak{a}} \backslash \Gamma \\\left|j\left(\sigma_{\mathfrak{a}}{ }^{-1} \gamma \sigma_{\mathfrak{a}}, \sigma_{\mathfrak{a}}{ }^{-1} z\right)\right|^{2} \leq T}} \Lambda(\gamma)=\frac{-T}{\operatorname{Im}\left(\sigma_{\mathfrak{a}}^{-1} z\right) \operatorname{Vol}(\Gamma \backslash \mathfrak{H})}\left(P_{\mathfrak{a}}(z, \Lambda)+\overline{Q_{\mathfrak{a}}(z, \Lambda)}\right)+O\left(T^{\frac{1}{2}+\epsilon}\right)
$$

as $T \rightarrow \infty$ where the implied constant depends on $z, \Lambda$ and $\Gamma$. The functions $P_{\mathfrak{a}}$ and $Q_{\mathfrak{a}}$ are as in proposition 5.1 .

Proof: We use the well known integral transform [Ap, p. 243], [Da]

$$
\frac{1}{2 \pi i} \int_{c-i Y}^{c+i Y} \frac{y^{s}}{s} d s=\delta(y)+\mathcal{E}(Y, y, c)
$$

\footnotetext{
${ }^{3}$ See the footnote in the introduction.
} 
where $c>0$,

$$
\delta(y)= \begin{cases}0 & \text { if } 0<y<1 \\ \frac{1}{2} & \text { if } y=1 \\ 1 & \text { if } y>1\end{cases}
$$

and the error $\mathcal{E}(Y, y, c)$ satisfies

$$
|\mathcal{E}(Y, y, c)| \leq \begin{cases}\pi^{-1} \frac{y^{c}}{Y|\log y|} & \text { if } y \neq 1 \\ \pi^{-1} \frac{c}{Y} & \text { if } y=1\end{cases}
$$

It follows that

$$
\begin{aligned}
& \sum_{\gamma \in \Gamma_{\mathfrak{a}} \backslash \Gamma} \delta\left(\frac{T}{\left|j\left(\sigma_{\mathfrak{a}}^{-1} \gamma \sigma_{\mathfrak{a}}, \sigma_{\mathfrak{a}}{ }^{-1} z\right)\right|^{2}}\right) \Lambda(\gamma)=\frac{1}{2 \pi i} \int_{2-i Y}^{2+i Y} E_{\mathfrak{a}}(z, s, \Lambda) \frac{T^{s}}{\operatorname{Im}\left(\sigma_{\mathfrak{a}}{ }^{-1} z\right)^{s}} \frac{d s}{s} \\
& -\sum_{\gamma \in \Gamma_{\mathfrak{a}} \backslash \Gamma} \mathcal{E}\left(Y, \frac{T}{\left|j\left(\sigma_{\mathfrak{a}}{ }^{-1} \gamma \sigma_{\mathfrak{a}}, \sigma_{\mathfrak{a}}^{-1} z\right)\right|^{2}}, 2\right) .
\end{aligned}
$$

We will later choose $Y$ to be approximately $T^{4}$. Shift the line of integration in the above integral to the line $\operatorname{Re}(s)=\frac{1}{2}+\epsilon$ for some small $\epsilon>0$. Since $E_{\mathfrak{a}}(z, s, \Lambda)$ is bounded for $\operatorname{Re}(s)>\frac{1}{2}+\epsilon$ (by Petridis [Pe, theorem 5]) it follows that the horizontal integrals are bounded by $O\left(T^{2} / Y\right)$ while the vertical integral on the line $\frac{1}{2}+\epsilon$ satisfies the bound

$$
\left|\frac{1}{2 \pi i} \int_{\frac{1}{2}+\epsilon-i Y}^{\frac{1}{2}+\epsilon+i Y} E_{\mathfrak{a}}(z, s, \Lambda) \frac{T^{s}}{\operatorname{Im}\left(\sigma_{\mathfrak{a}}^{-1} z\right)^{s}} \frac{d s}{s}\right| \ll T^{\frac{1}{2}+\epsilon} \log Y .
$$

The residue of the pole at $s=1$ gives the main term in theorem 7.3. To deal with the error $\operatorname{sum} \sum_{\gamma \in \Gamma_{\mathfrak{a}} \backslash \Gamma} \mathcal{E}\left(Y, \frac{T}{\left|j\left(\sigma_{\mathfrak{a}}^{-1} \gamma \sigma_{\mathfrak{a}}, \sigma_{\mathfrak{a}}-1 z\right)\right|^{2}}, 2\right)$ we break up $\Gamma_{\mathfrak{a}} \backslash \Gamma$ into three subsets:

$$
\begin{aligned}
& S_{1}(T)=\left\{\gamma \in \Gamma_{\mathfrak{a}} \backslash \Gamma \mid \frac{T}{\left|j\left(\sigma_{\mathfrak{a}}^{-1} \gamma \sigma_{\mathfrak{a}}, \sigma_{\mathfrak{a}}{ }^{-1} z\right)\right|^{2}}=1\right\}, \\
& S_{2}(T)=\left\{\gamma \in \Gamma_{\mathfrak{a}} \backslash \Gamma \mid \frac{T}{\left|j\left(\sigma_{\mathfrak{a}}^{-1} \gamma \sigma_{\mathfrak{a}}, \sigma_{\mathfrak{a}}{ }^{-1} z\right)\right|^{2}} \in\left(0, e^{-1}\right] \cup[e, \infty)\right\}, \\
& S_{3}(T)=\left\{\gamma \in \Gamma_{\mathfrak{a}} \backslash \Gamma \mid \frac{T}{\left|j\left(\sigma_{\mathfrak{a}}-1 \gamma \sigma_{\mathfrak{a}}, \sigma_{\mathfrak{a}}-1 z\right)\right|^{2}} \in\left(e^{-1}, 1\right) \cup(1, e)\right\} .
\end{aligned}
$$

In order to get some control on the size of the above sets we use the following lemma.

Lemma 7.4. For any Fuchsian group of the first kind $\Gamma$ and any $z$ in $\mathfrak{H}$ there exists a constant $C=C(\Gamma, z)$ so that for any $T \gg 1$ we have

$$
\#\left\{\gamma \in \Gamma_{\mathfrak{a}} \backslash \Gamma|| \log \left(T \cdot \operatorname{Im}\left(\sigma_{\mathfrak{a}}{ }^{-1} \gamma z\right)\right) \mid \leq C\right\} \ll \log T
$$


with the implied constant depending only on $\Gamma$ and $z$.

The proof uses the hyperbolic geometry of $\mathfrak{H}$ and we postpone it until the end of this section. For now we just need to know that the hyperbolic distance between $z, w$ in $\mathfrak{H}$ is

$$
\rho(z, w)=\log \frac{|z-\bar{w}|+|z-w|}{|z-\bar{w}|-|z-w|} .
$$

See [Iw, chapter 1] for example. This implies that $\rho(i A, i B)=|\log (A / B)|$ for $A, B>0$. We shall need the following equivalent formulations of lemma 7.4:

$$
\begin{aligned}
& \#\left\{\gamma \in \Gamma_{\mathfrak{a}} \backslash \Gamma|| \log \left(T\left|j\left(\sigma_{\mathfrak{a}}{ }^{-1} \gamma \sigma_{\mathfrak{a}}, \sigma_{\mathfrak{a}}{ }^{-1} z\right)\right|^{-2}\right) \mid \leq C\right\} \ll \log T, \\
& \#\left\{\gamma \in \Gamma_{\mathfrak{a}} \backslash \Gamma \mid \rho\left(\frac{i}{T}, i \operatorname{Im}\left(\sigma_{\mathfrak{a}}{ }^{-1} \gamma z\right)\right) \leq C\right\} \ll \log T,
\end{aligned}
$$

where $C=C(\Gamma, z)$ as before.

It is now clear that $S_{1}$ has $O(\log T)$ elements and thus

$$
\sum_{\gamma \in S_{1}(T)} \mathcal{E}\left(Y, \frac{T}{\left|j\left(\sigma_{\mathfrak{a}}^{-1} \gamma \sigma_{\mathfrak{a}}, \sigma_{\mathfrak{a}}^{-1} z\right)\right|^{2}}, 2\right) \ll \frac{\log T}{Y} .
$$

Also if $y \in\left(0, e^{-1}\right] \cup[e, \infty)$ we have $|\log y|^{-1} \leq 1$ so that

$$
\sum_{\gamma \in S_{2}(T)} \mathcal{E}\left(Y, \frac{T}{\left|j\left(\sigma_{\mathfrak{a}}^{-1} \gamma \sigma_{\mathfrak{a}}, \sigma_{\mathfrak{a}}^{-1} z\right)\right|^{2}}, 2\right) \ll \frac{T^{2}}{Y} E_{\mathfrak{a}}(z, 2) \ll \frac{T^{2}}{Y} .
$$

The sum over $\gamma$ in $S_{3}$ can be large if $T\left|j\left({\sigma_{\mathfrak{a}}}^{-1} \gamma{\sigma_{\mathfrak{a}}},{\sigma_{\mathfrak{a}}}^{-1} z\right)\right|^{-2} \sim 1$. We replace $T$ by $U$ so that this expression is bounded away from 1. Precisely, by (7.5), there exists a $U$ with

$$
\rho\left(\frac{i}{T}, \frac{i}{U}\right) \leq \frac{C}{T}
$$

such that for all $\gamma \in \Gamma_{\mathfrak{a}} \backslash \Gamma$ we have

$$
\rho\left(\frac{i}{U}, i \operatorname{Im}\left(\sigma_{\mathfrak{a}}{ }^{-1} \gamma z\right)\right) \gg \frac{C}{T \log T} .
$$

Note that (7.6) implies that $|T-U| \ll C \ll 1$ and (7.7) implies that

$$
\left|\log \left(\frac{U}{\left|j\left(\sigma_{\mathfrak{a}}^{-1} \gamma \sigma_{\mathfrak{a}}, \sigma_{\mathfrak{a}}{ }^{-1} z\right)\right|^{2}}\right)\right|^{-1} \ll T \log T \ll U \log U
$$

for all $\gamma$ in $\Gamma_{\mathfrak{a}} \backslash \Gamma$. Therefore,

$$
\sum_{\gamma \in S_{3}(U)} \mathcal{E}\left(Y, \frac{U}{\left|j\left(\sigma_{\mathfrak{a}}^{-1} \gamma \sigma_{\mathfrak{a}}, \sigma_{\mathfrak{a}}^{-1} z\right)\right|^{2}}, 2\right) \ll \frac{U^{3} \log U}{Y} E_{\mathfrak{a}}(z, 2) \ll \frac{U^{3} \log U}{Y} .
$$


Assembling these results we have demonstrated that for large $T$ we may find $U$ within a bounded distance of $T$ so that

$$
\begin{gathered}
\sum_{\gamma \in \Gamma_{\mathfrak{a}} \backslash \Gamma} \delta\left(\frac{U}{\left|j\left(\sigma_{\mathfrak{a}}{ }^{-1} \gamma \sigma_{\mathfrak{a}}, \sigma_{\mathfrak{a}}{ }^{-1} z\right)\right|^{2}}\right) \Lambda(\gamma)=\frac{-U}{\operatorname{Im}\left(\sigma_{\mathfrak{a}}{ }^{-1} z\right) \operatorname{Vol}(\Gamma \backslash \mathfrak{H})}\left(P_{\mathfrak{a}}(z, \Lambda)+\overline{Q_{\mathfrak{a}}(z, \Lambda)}\right) \\
+O\left(U^{\frac{1}{2}+\epsilon}\right)
\end{gathered}
$$

where we chose $Y=U^{4}$. Now replacing $U$ by $T$ on the left-hand side of (7.8) introduces $O(\log T)$ extra terms in the sum by $(7.5),(7.6)$. From section 2 we have

$$
\Lambda(\gamma) \ll \operatorname{Im}\left(\sigma_{\mathfrak{a}}^{-1} \gamma z\right)^{-R_{\Lambda}-\varepsilon} \ll T^{R_{\Lambda}+\varepsilon}
$$

For $\Lambda$ cuspidal and $\Gamma$ a congruence group we have $R_{\Lambda}=1 / 2$. Thus

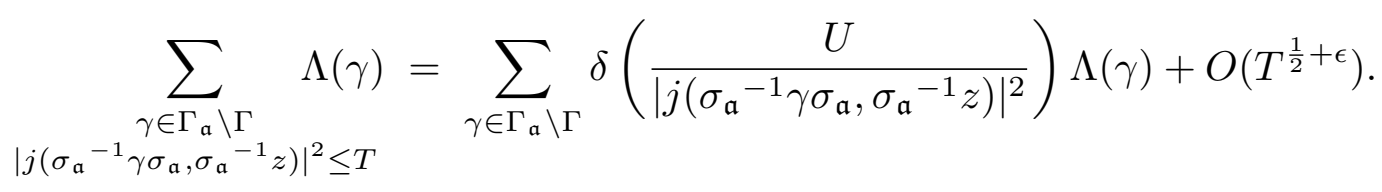

Finally, replacing $U$ by $T$ on the right of (7.8) introduces only a bounded constant and we obtain the theorem.

It only remains to prove the lemma. Recall the fundamental domain $\mathcal{F} \subset \mathcal{F}_{\infty}$ introduced in proposition 2.5. We simplify the exposition by replacing the cusp $\mathfrak{a}$ by $\infty$. Take $z$ in the interior of $\mathcal{F}$. Choose a radius $2 C$ so that the hyperbolic ball centered at $z$ of this radius (denoted $\mathcal{B}(z, 2 C)$ ) is also contained in $\mathcal{F}$. Since $\Gamma$ acts by isometries on the hyperbolic space $\mathfrak{H}$ we have

$$
\bigcup_{\gamma \in \Gamma_{\infty} \backslash \Gamma} \mathcal{B}(\gamma z, 2 C) \subset \mathcal{F}_{\infty}
$$

a disjoint union. This observation already leads to an elegant proof of the absolute convergence of $E(z, s)$ for $\operatorname{Re}(s)>1$, see [Sa, $\S 1.4]$.

If we look at the horizontal line segment $L=[-1 / 2+i / T, 1 / 2+i / T]$ in $\mathcal{F}_{\infty}$ we see that any $\gamma$ in $\Gamma_{\infty} \backslash \Gamma$ with $\rho\left(\frac{i}{T}, i \operatorname{Im}\left(\sigma_{\infty}{ }^{-1} \gamma z\right)\right) \leq C$ must have $\mathcal{B}(\gamma z, 2 C)$ intersecting $L$. The hyperbolic length of $L$ is $\log \left(1+\frac{2}{-1+\sqrt{4 T^{-2}+1}}\right) \ll \log T$ so

$$
\#\left\{\gamma \in \Gamma_{\infty} \backslash \Gamma \mid \rho\left(\frac{i}{T}, i \operatorname{Im}\left(\sigma_{\infty}^{-1} \gamma z\right)\right) \leq C\right\} \ll \frac{\log T}{C} \ll \log T
$$

establishing (7.5) as required.

\section{Application to $\Gamma_{0}(4)$.}

Let $\Gamma=\Gamma_{0}(4) /\{ \pm 1\}$ be the image of the Hecke congruence group of level 4 in $P S L_{2}(\mathbb{Z})$. Then $\Gamma \backslash \mathfrak{H}$ has genus $g=0$, no elliptic fixed points and $m=3$ cusps which we may take to be $\infty, 0,1 / 2$. Their stability groups are $\Gamma_{\infty}, \Gamma_{0}$ and $\Gamma_{1 / 2}$ which are generated by

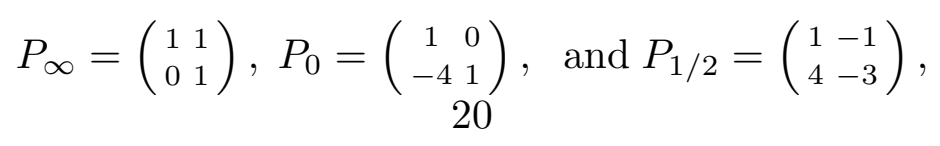


respectively, and we may take $\sigma_{\infty}=\left(\begin{array}{ll}1 & 0 \\ 0 & 1\end{array}\right), \sigma_{0}=\left(\begin{array}{cc}0 & -1 / 2 \\ 2 & 0\end{array}\right)$ and $\sigma_{1 / 2}=\left(\begin{array}{ll}1 & 0 \\ 2 & 1\end{array}\right)$.

Also $\Gamma$ is generated by these three elements with the single relation $P_{\infty} P_{0} P_{1 / 2}=1$. So, in effect, $\Gamma$ is freely generated by two of these, say $P_{\infty}$ and $P_{0}$. Define $\Lambda_{\infty}, \Lambda_{0}$ so that $\Lambda_{\infty}\left(P_{\infty}\right)=\Lambda_{0}\left(P_{0}\right)=0$ and $\Lambda_{\infty}\left(P_{0}\right)=\Lambda_{0}\left(P_{\infty}\right)=1$. Also set $\Lambda_{1 / 2}=\Lambda_{\infty}-\Lambda_{0}$. Then, for each cusp $\mathfrak{a}, \mathcal{V}_{\mathfrak{a}}(\Gamma)$ is one dimensional and we may take the basis element to be $\Lambda_{\mathfrak{a}}$.

Since $g=0$ there are no weight 2 cusp forms but $\mathcal{M}_{2}(\Gamma)$ is 2 dimensional. A basis may be found as in section 4 . It is simpler in this case to use a holomorphic function $G_{2}(z)$ that almost transforms as a weight two function on the full modular group,

$$
G_{2}(z)=\frac{-1}{24}+\sum_{n=1}^{\infty} \sigma(n) e^{2 \pi i n z}
$$

where $\sigma(n)=\sum_{d \mid n} d$. This is described for example in [Za]. It turns out that $f_{1}(z)=$ $G_{2}(z)-2 G_{2}(2 z)$ and $f_{2}(z)=G_{2}(z)-4 G_{4}(4 z)$ are two linearly independent modular forms for $\Gamma_{0}(4)$. The constant terms in the Fourier expansions of $f_{1}$ and $f_{2}$ at $\infty$ are $1 / 24$ and $1 / 8$, respectively. For the expansion of $f_{1}$ at the cusp 0 we need to calculate

$$
j\left(\sigma_{0}, z\right)^{-2} f_{1}\left(\sigma_{0} z\right)=(2 z)^{-2}\left(G_{2}\left(\sigma_{0} z\right)-2 G_{2}\left(2 \sigma_{0} z\right)\right) .
$$

If $S=\left(\begin{array}{cc}0 & -1 \\ 1 & 0\end{array}\right)$ then we know [Za] that $G_{2}(S z)=j(S, z)^{2} G_{2}(z)-j(S, z) /(4 \pi i)=z^{2} G_{2}(z)-$ $z /(4 \pi i)$. Also $\sigma_{0} z=S(4 z)$ and $2 \sigma_{0} z=S(2 z)$ so we obtain

$$
\begin{aligned}
G_{2}\left(\sigma_{0} z\right)-2 G_{2}\left(2 \sigma_{0} z\right) & =G_{2}(S(4 z))-2 G_{2}(S(2 z)) \\
& =(4 z)^{2} G_{2}(4 z)-2(2 z)^{2} G_{2}(2 z)
\end{aligned}
$$

and $j\left(\sigma_{0}, z\right)^{-2} f_{1}\left(\sigma_{0} z\right)=4 G_{2}(4 z)-2 G_{2}(2 z)$. Similarly $j\left(\sigma_{0}, z\right)^{-2} f_{2}\left(\sigma_{0} z\right)=4 G_{2}(4 z)-G_{2}(z)$. The constant terms of $f_{1}$ and $f_{2}$ at 0 are $-1 / 12$ and $-1 / 8$. Thus, if we set

$$
f_{\infty}=24 f_{1}-8 f_{2}, f_{0}=-24 f_{1}+16 f_{2}
$$

then the constant terms of $f_{\infty}$ (and $\left.f_{0}\right)$ at the cusps $\infty, 0,1 / 2$ are $0,1,-1$ (and $\left.1,0,-1\right)$, respectively.

By proposition 2.1 we know that $\Lambda_{\infty}$ is a linear combination of the real and imaginary parts of the modular symbols $\left\langle\gamma, f_{\infty}\right\rangle,\left\langle\gamma, f_{0}\right\rangle$. We must have $p_{\Lambda_{\infty}}=f_{\infty} / 2=q_{\Lambda_{\infty}}$ and $\Lambda_{\infty}(\gamma)=\left\langle\gamma, f_{\infty}\right\rangle / 2+\overline{\left\langle\gamma, f_{\infty}\right\rangle} / 2$ where

$$
f_{\infty}(z)=16 \sum_{n \geq 1, n \text { odd }} \sigma(n) e^{2 \pi i n z}
$$

Therefore,

$$
P_{\infty}\left(z, \Lambda_{\infty}\right)=Q_{\infty}\left(z, \Lambda_{\infty}\right)=\int_{\infty}^{z} f_{\infty}(w) / 2 d w=\frac{8}{2 \pi i} \sum_{n \geq 1, n \text { odd }} \frac{\sigma(n)}{n} e^{2 \pi i n z}
$$


Theorem 7.1 implies that

$$
\begin{gathered}
J_{\infty}\left(z, T, \Lambda_{\infty}\right)=\sum_{\substack{\gamma \in \Gamma_{\infty} \backslash \Gamma \\
|j(\gamma, z)|^{2}<T}} \Lambda_{\infty}(\gamma)\left(1-\frac{|j(\gamma, z)|^{2}}{T}\right) \\
\ll_{\varepsilon}\left(y^{-3 / 2-\varepsilon}+y^{-15 / 4-\varepsilon}\right) T^{5 / 4+\varepsilon} .
\end{gathered}
$$

Theorem 7.2 (depending on conjecture 1.1) implies

$$
\begin{aligned}
J_{\infty}\left(z, T, \Lambda_{\infty}\right) & =\frac{-T}{2 y \operatorname{Vol}(\Gamma \backslash \mathfrak{H})}\left(P_{\infty}\left(z, \Lambda_{\infty}\right)+\overline{Q_{\infty}\left(z, \Lambda_{\infty}\right)}+U_{\infty}\left(z, \Lambda_{\infty}\right)\right)+O\left(T^{1-\varepsilon}\right) \\
& =\frac{-2 T}{\pi^{2} y}\left(\sum_{n \geq 1, n \text { odd }} \frac{\sigma(n)}{n} e^{-2 \pi n y} \sin (2 \pi n x)+\frac{\pi}{8} U_{\infty}\left(z, \Lambda_{\infty}\right)\right)+O\left(T^{1-\varepsilon}\right),
\end{aligned}
$$

where $U_{\infty}\left(z, \Lambda_{\infty}\right)$ has yet to be determined. The result for $J_{0}\left(\sigma_{0} z, T, \Lambda_{0}\right)$ is similar.

We conclude this section by giving two more proofs of the fact that $F(z)=P_{\infty}\left(z, \Lambda_{\infty}\right)+$ $\overline{Q_{\infty}\left(z, \Lambda_{\infty}\right)}$ has the correct transformation property when $z \rightarrow P_{0} z=\frac{z}{-4 z+1}$,

$$
F\left(P_{0} z\right)-F(z)=\Lambda_{\infty}\left(P_{0}\right)=1
$$

Our justification is that these proofs are independently interesting and may also prove valuable in extending our ideas as we shall see in the next section.

Note that $F\left(P_{0} z\right)-F(z)$ is independent of $z$. Instead of examining $F$ near the cusp $z=0$ we take $z=(-1+i) / 4$. Then $P_{0} z=(1+i) / 4$ and

$$
F\left(P_{0} z\right)-F(z)=\frac{16}{\pi} \sum_{n} \frac{\sigma(n)}{n} e^{-2 \pi n / 4} \sin (\pi n / 2) .
$$

Lemma 8.1. We have

$$
\sum_{n=1}^{\infty} \frac{\sigma(n)}{n} e^{\frac{-\pi n}{2}} \chi(n)=\frac{\pi}{16}
$$

where $\chi(n)=\sin (\pi n / 2)$ is zero unless $n$ is odd, in which case $\chi(n)=(-1)^{\frac{n-1}{2}}$.

Proof: Define the Dirichlet $L$-function

$$
L(s, \chi)=\sum_{n=1}^{\infty} \frac{\chi(n)}{n^{s}}
$$

Then

$$
L(s, \chi) L(s+1, \chi)=\sum_{n} \frac{\chi(n)}{n^{s}} \sum_{d \mid n} \frac{1}{d}
$$


and we obtain

$$
\frac{1}{2 \pi i} \int_{(2)} \frac{\Gamma(s)}{(\pi / 2)^{s}} L(s, \chi) L(s+1, \chi) d s=\sum_{n=1}^{\infty} \frac{\sigma(n)}{n} e^{\frac{-\pi n}{2}} \chi(n),
$$

where we integrate along the line $\operatorname{Re}(s)=2$. To evaluate this integral we employ the functional equation for $L$ :

$$
\left(\frac{4}{\pi}\right)^{\frac{s}{2}} \Gamma\left(\frac{s+1}{2}\right) L(s, \chi)=\left(\frac{4}{\pi}\right)^{\frac{1-s}{2}} \Gamma\left(\frac{2-s}{2}\right) L(1-s, \chi)
$$

and the duplication formula to get

$$
\begin{aligned}
\xi(s) & =\left(\frac{2}{\pi}\right)^{s} \frac{\Gamma(s+1)}{s} L(s, \chi) L(s+1, \chi) \\
& =-\left(\frac{2}{\pi}\right)^{-s} \frac{\Gamma(1-s)}{-s} L(-s, \chi) L(1-s, \chi)=-\xi(-s) .
\end{aligned}
$$

Moving the line of integration to $\operatorname{Re}(s)=-2$ we pick up the residue of $\xi$ at $s=0$,

$$
\frac{1}{2 \pi i} \int_{(2)} \xi(s) d s=\frac{1}{2} \operatorname{Res}_{s=0} \xi(s)=\frac{1}{2} L(0, \chi) L(1, \chi)=\frac{L(1, \chi)^{2}}{\pi}=\frac{\pi}{16} .
$$

We used the fact that $L(1, \chi)=\frac{\pi}{4}$, see [Da].

It was pointed out to us by Bruce Berndt that lemma 8.1 also follows quickly from a result of Ramanujan. Part of entry 15 in chapter 14 of Ramanujan's second notebook [Be1, pp. 262-263] gives

$$
\sum_{n=1}^{\infty} \chi(n) \frac{\operatorname{sech}(\alpha n)}{n}+\sum_{n=1}^{\infty} \chi(n) \frac{\operatorname{sech}(\beta n)}{n}=\pi / 4
$$

for $\alpha, \beta>0$ with $\alpha \beta=\pi^{2} / 4$. To derive lemma 8.1 from this observe that

$$
\begin{aligned}
\frac{1}{2} \sum_{n=1}^{\infty} \chi(n) \frac{\operatorname{sech}(n \pi / 2)}{n} & =\sum_{n=1}^{\infty} \chi(n) \frac{e^{-n \pi / 2}}{n\left(1+e^{-n \pi}\right)} \\
& =\sum_{n=1}^{\infty} \chi(n) \frac{e^{-n \pi / 2}}{n} \sum_{m=0}^{\infty}(-1)^{m} e^{-m n \pi} \\
& =\sum_{n=1}^{\infty} \frac{\chi(n)}{n} \sum_{m=0}^{\infty}(-1)^{m} e^{-(2 m+1) n \pi / 2} \\
& =\sum_{n=1}^{\infty} \frac{\chi(n)}{n} \sum_{m=1}^{\infty} \chi(m) e^{-m n \pi / 2} \\
& =\sum_{r=1}^{\infty} \sum_{n \mid r} \frac{1}{n} \chi(r) e^{-r \pi / 2} \\
& =\sum_{r=1}^{\infty} \frac{\sigma(r)}{r} \chi(r) e^{-r \pi / 2} .
\end{aligned}
$$


The lemma now follows from (8.3) with $\alpha=\beta=\pi / 2$. The first published proof of (8.3) appears in [Ma]. A proof in [Be2, prop. 4.5] which generalizes (8.3), and may be used to deduce a wide class of interesting identities, comes from the transformation formula of the generalized Eisenstein series

$$
G\left(z, s ; \chi_{1}, \chi_{2} ; r_{1}, r_{2}\right)=\sum_{m, n=-\infty}^{\infty} \frac{\chi_{1}(m) \chi_{2}(n)}{\left(\left(m+r_{1}\right) z+n+r_{2}\right)^{s}}
$$

as $z$ goes to $\gamma z$ for $\gamma$ in $\Gamma_{0}(1)$. Here $\chi_{1}, \chi_{2}$ are primitive characters of modulus $k$, the numbers $r_{1}, r_{2}$ are arbitrary reals and $z$ is in $\mathfrak{H}$. Also $\operatorname{Re}(s)>2$ initially but $G$ may be analytically continued to all $s$ in $\mathbb{C}$. The above sum excludes $m=-r_{1}$ and $n=-r_{2}$.

To prove (8.3) Berndt uses the special case $\chi_{1}=\chi_{2}=\chi, r_{1}=r_{2}=0, s=0$ and $z=\frac{\pi i}{2 \alpha}$ mapping to $\frac{-1}{z}=\frac{\pi i}{2 \beta}$. See $[\mathrm{Be} 2]$.

\section{Application to $\Gamma_{0}(11)$.}

For $\Gamma=\Gamma_{0}(11) /\{ \pm 1\}$ the Riemann surface corresponding to $\Gamma \backslash \mathfrak{H}$ has genus $g=1$. For generators we may take

$$
A=\left(\begin{array}{cc}
-7 & -1 \\
22 & 3
\end{array}\right), B=\left(\begin{array}{cc}
4 & 1 \\
-33 & -8
\end{array}\right), P_{0}=\left(\begin{array}{cc}
1 & 0 \\
-11 & 1
\end{array}\right), P_{\infty}=\left(\begin{array}{ll}
1 & 1 \\
0 & 1
\end{array}\right)
$$

and they satisfy the relation $A B A^{-1} B^{-1} P_{0} P_{\infty}=1$ as in the description at the start of section 2. We have $m=2$ cusps at $\infty$ and 0 . Set $\sigma_{\infty}=\left(\begin{array}{ll}1 & 0 \\ 0 & 1\end{array}\right)$ and $\sigma_{0}=\left(\begin{array}{cc}0 & -1 / \sqrt{11} \\ \sqrt{11} & 0\end{array}\right)$.

The dimensions of $\mathcal{V}(\Gamma), \mathcal{V}_{0}(\Gamma), \mathcal{V}_{\infty}(\Gamma)$ and $\mathcal{W}(\Gamma)$ are 3,2,2 and 2, respectively. We may take basis elements of these spaces to be $\Lambda_{A}, \Lambda_{B}$ and $\Lambda_{\infty}$ where $\Lambda_{A}=\log _{A}, \Lambda_{B}=\log _{B}$ and $\Lambda_{\infty}$ satisfies $\Lambda_{\infty}(A)=\Lambda_{\infty}(B)=0$ and $\Lambda_{\infty}\left(P_{\infty}\right)=-\Lambda_{\infty}\left(P_{0}\right)=1$. (Recall our definition of $\log$ from section 1.)

The space $\mathcal{M}_{2}(\Gamma)$ contains the Eisenstein series $g(z)=G_{2}(z)-11 G_{2}(11 z)$ and the newform $f(z)=\sqrt[12]{\Delta(z) \Delta(11 z)}=\eta(z)^{2} \eta(11 z)^{2}$ for $\Delta$ the discriminant function and $\eta$ Dedekind's eta function. The Fourier expansions of $g$ and $f$ are

$$
\begin{aligned}
& g(z)=\frac{5}{12}+\sum_{n=1}^{\infty} \sum_{\substack{d \mid n \\
11 \nmid d}} d e^{2 \pi i n z}, \\
& f(z)=\sum_{a, b, c, d \geq 1} \chi(a b c d) e^{2 \pi i z\left(\frac{a^{2}+b^{2}+11\left(c^{2}+d^{2}\right)}{24}\right)},
\end{aligned}
$$

where $\chi$ is a primitive character defined by

$$
\chi(n)=\left\{\begin{aligned}
1 & \text { for } n \equiv \pm 1(12) \\
-1 & \text { for } n \equiv \pm 5(12) \\
0 & \text { otherwise }
\end{aligned}\right.
$$


The formula (9.2) follows directly from Ramanujan's expression for the Dedekind eta function

$$
\eta(z)=\sum_{n=1}^{\infty} \chi(n) e^{2 \pi i z\left(\frac{n^{2}}{24}\right)},
$$

which, in turn, may be proved using the Jacobi triple product formula [Bu, p. 29]. It can be seen that $\left\langle P_{\infty}, g\right\rangle=5 / 12=-\left\langle P_{0}, g\right\rangle$ and $\left\langle P_{\infty}, f\right\rangle=\left\langle P_{0}, f\right\rangle=0$. The values of $\langle A, f\rangle,\langle B, f\rangle,\langle A, g\rangle$ and $\langle B, g\rangle$ are not as easy to find. Using (9.1), (9.2) their values may be computed to arbitrary precision. It would be interesting to find them exactly, perhaps using ideas from the proofs of lemma 8.1. We find (see also [Kna, (11.6)]) that $\langle A, f\rangle=u+v i=-\overline{\langle B, f\rangle}$ where, approximately,

$$
u+v i=-0.23217787565035+0.10100046729715 i .
$$

Employing proposition 2.1 as in the last section we have $p_{\Lambda_{A}}=q_{\Lambda_{A}}=\left(\frac{1}{4 u}-\frac{i}{4 v}\right) f$ and

$$
\begin{aligned}
\operatorname{Res}_{s=1} E_{\infty}\left(z, s, \Lambda_{A}\right) & =-\operatorname{Vol}(\Gamma \backslash \mathfrak{H})^{-1}\left(P_{\infty}\left(z, \Lambda_{A}\right)+\overline{Q_{\infty}\left(z, \Lambda_{A}\right)}\right) \\
& =\frac{-1}{2 \pi} \operatorname{Re}\left(\left(\frac{1}{4 u}-\frac{i}{4 v}\right) \int_{\infty}^{z} f(w) d w\right)
\end{aligned}
$$

yielding theorem 1.2. Similarly $p_{\Lambda_{B}}=q_{\Lambda_{B}}=-\left(\frac{1}{4 u}+\frac{i}{4 v}\right) f$.

Acknowledgment. Both authors would like to thank Bruce Berndt for his elegant proof of lemma 8.1 .

\section{REFERENCES}

[Ap] T. Apostol, Introduction to Analytic Number Theory, Springer-Verlag, 1975.

[Be1] B. Berndt, Ramanujan's Notebooks. Part II, Springer-Verlag, New York, 1989.

[Be2] B. Berndt, Modular transformations and generalizations of several formulae of Ramanujan, Rocky Mt. J. Math. 7 (1977), 147-189.

[Bu] D. Bump, Automorphic Forms and Representations, Cambridge Studies in Advanced Mathematics 55, 1997.

[Ch-Go] G. Chinta, D. Goldfeld, Grössencharakter L-functions of real quadratic fields twisted by modular symbols, Invent. Math 144 (2001), no. 3, 435-449.

[Ch-O'S] G. Chinta, C. O'Sullivan, Non-holomorphic Poincaré series constructed from derivatives and antiderivatives of cusp forms and bounds on period polynomials (to appear).

[Da] H. Davenport, Multiplicative Number Theory, Berlin-Heidelberg-New York: Springer, 1980.

[Di-O'S] N. Diamantis, C. O'Sullivan, Hecke Theory of Series formed with Modular Symbols and Relations among Convolution L-functions, Math. Ann. 318 (2000), no. 1, 85-105.

[F-K] R.Fricke, F.Klein, Vorlesungen über die Theorie der automorphen Funktionen I,II., Leipzig, 1897, 1912.

[Go1] D. Goldfeld, The distribution of modular symbols, Number Theory in Progress (Proceedings of the International Conference organized by the S. Banach Intern. Math. Center in honor of Schinzel in Zakopane, Poland, June 30-July 9, 1997) (1999).

[Go2] D. Goldfeld, Zeta functions formed with modular symbols, Proc. of Symposia in Pure Math. 66.1 (1999), 111-121.

[Good] A. Good, Cusp Forms and Eigenfunctions of the Laplacian, Math. Ann. 255 (1981), 523-548. 
[He] D. A. Hejhal, The Selberg Trace Formula for $P S L(2, \mathbb{R})$, Lecture Notes in Math. 1001 (1983), Springer.

[Iw1] H. Iwaniec, Introduction to the Spectral Theory of Automorphic Forms, Bibl. Rev. Mat. Iber., Madrid, 1995.

[Iw2] H. Iwaniec, Topics in classical automorphic forms, vol. 17, Graduate studies in Math., American Math. Soc., 1997.

[Kna] A. W. Knapp, Elliptic curves, Princeton Univ. Press, 1992.

[Kno] M. Knopp, Some new results on the Eichler cohomology of automorphic forms, Bull. of the AMS 80 (1974), 607-632.

[Ma] S. L. Malurkar, On the application of Herr Mellin's integrals to some series, J. Indian Math. Soc. 16 (1925-1926), 130-138.

[O'S1] C. O'Sullivan, Properties of Eisenstein Series formed with Modular Symbols, J. reine angew. Math 518 (2000), 163-186.

[O'S2] C. O'Sullivan, Ph.D. Thesis, Columbia University (1998).

[Pe] Y. Petridis, Spectral Deformations and Eisenstein Series associated with Modular Symbols, Int. Math. Res. Not. 19 (2002), 991-1006.

[Pe-Ri] Y. Petridis, M. S. Risager, Distribution of modular symbols and Eisenstein series (in preparation).

[Ri] M. S. Risager, Ph.D. Thesis, University of Aarhus (2003).

[Sa] P. Sarnak, Some Applications of Modular Forms, Cambridge Tracts in Math. 99, Cambridge Univ. Press, 1990.

[Sc] B. Schoeneberg, Elliptic modular functions, Springer Verlag, 1974.

[Se] A. Selberg, Harmonic Analysis, in Collected Papers vol. I (1989), Springer.

[Sh] G. Shimura, Introduction to the Arithmetic Theory of Automorphic Functions, Princeton Univ. Press, 1971.

[Za] D. Zagier, Introduction to modular forms (1992), From number theory to physics (Les Houches, 1989) Springer, Berlin, 238-291.

Dorian Goldfeld

Columbia University

Department of Mathematics

New York, NY 10027

goldfeld@columbia.edu
Cormac O'Sullivan

Bronx Community College

of the City University of New York

Dept. of Math \& Computer Science

University Ave. at West 181 St.

Bronx, NY 10453

CO’Sullivan@gc.cuny.edu 